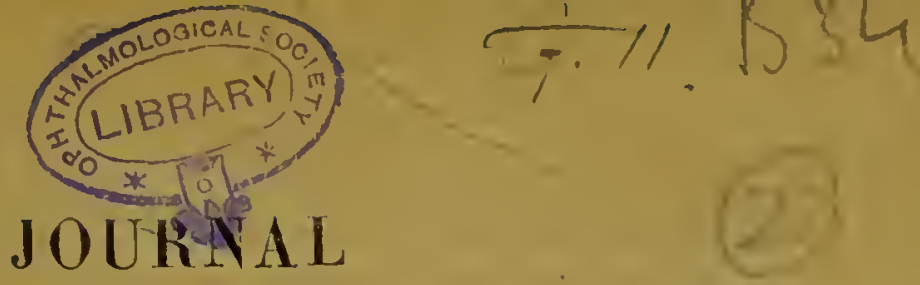

\title{
$\mathrm{DE}$ \\ L'A N A TOMIE \\ E T DE
}

\section{LA PHYSIOLOGIE}

NORMALES ET PATHOLOGIQUES

DE L'HONME ET DES ANIMAUX

DE

\section{LA RÉGÉNÉRATION DU GRISTALLIN}

GHEZ QUELQUES MAMMIE ĖRS

Par le $\mathbf{D}^{\mathrm{r}}$ Renjamin MLLIOT.

PLANGHES I, $11,111,1 \mathrm{~V}, \mathrm{~V}$ ET VI.

\section{PRÉLIMINAIRES.}

La monographie que nous publions aujourd'hui est un travail de longue lialeinc. Il est le fruit des recherches que nous avons entreprises encore en 1863, à Kiew (Russie), d'après le conseil de notrc vénérable maitre, M. W. Karavaïcw, et que malheureusement diverses circonstances de la vie ne nous ont pas permis de poursuivre avec plus d'assiduité : le lecteur y aurait trouvé moins de lacures et nous plus d'une question secondaire rćsolue au profit de la science, à cóté de celle qui les domine toutes, la régénération du eristallin normal chez quelques manmifères. Nrus avions l'intention de publier nos recherehes beaucoup plus larrl, cspérant pouvoir trancher du mème coup l'importante ruestion, iu point de vue pralique, de la régénération ou de la non-régénération dı cristallin chez l'homme après la cataractoSOIRS, IE, L'ANAT. ET DE I,A PHYSIOL. - $\mathrm{r}$, VIII (1872). 
tonir, mais les recentes recherelies de M. Philiperux sont venues, en quelque sorte, nons forcer la main.

Lorsqu'en 1867 nous présentâmes à l'Académie des sciences de Paris notre inćmoirc sur la régénération du cristallin (1), el - lorsqu'en 1868 nous publiâmes en russe un travail sur le méme sujet (2), nons étions loin de penser que des faits étudiés avec patience et impartialité, ct liv'és comme tels ì la publicité, pussent élever des doules chez des confrères à esprit judicieux et ì âme bien néc. La chose cut licu cependant, el ce ne fut que plus tard seulement, quand, passionné pour la lutte avec les difficultés de notre art, nous nous allaquàmes résolument ì des questions ardues el en amont du courant scicntifique, que nous com. prîmes les déceptions inhérentes à loute recherche de progrès dans là sricnce.

Nous constatons avec, bonheur que le professcur W. Karavaïew, de Kiew, fut de ceux qui ne doutérent ni de la bonne foi de nos travaux mentionnés, ni des faits qui s'y trouvaient consignés : il savait que ses élèves élaient incapables de s'engager dars celte nouvclle voic médicale, créée en dehor's de nos traditions par des esprits d'innovation témérairc, el acclamée par les naiures moutonnières ou paresscuses, oì l'analyse écœurante n'est plus équilibrée par la synthèse et oủ la négation est devenue la base des recherches les plus iusignifiantes et des critiques les moins partiales.

Pour nous faire mieux comprendre, il suffira de dire que l'écolc allemande actuclle, essentiellement analytique, et ses dérivís, partis du nililisme de l'école de Viemne cl trainés par leur puissant remorqueur, Virchow, sont arrivés à remplacer l'irritation des vaisseaux par celle des cellules, à trouver le type de l'inflammation

(1) B. Milliot, Mémoire sur la régénération du cristallin (Comples rendus do l'Academie des seienees, 28 janvier 1867).

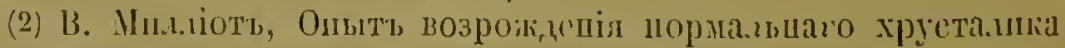

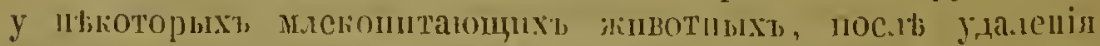

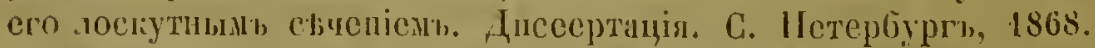
B. Milliot, Essai sur la régénération du cristallin chez quelques mammifères après l'extraction du cristallin normal par la lidralolomic (thèse inaugurale). Saint-Petersbourg, 1868. 
daus les tissus non vasculaires (cornce et cartilages), à regarder comme certainc la provenance des éléments anatomiques par scission répétéc (prolifération) des éléments analomiques-souches d'espécc différente; bref ì bouleverser nos notions les plus élémentaires de pathologie, à rompre avec nos traditions cliniqucs et il poursuiyrc le sentier étroit de la nćgation (1).

Le lccteur trouvera dans notre monographie des indications bibliographiques sur la régénération du cristallin plus étendues qu'il n'eût été à même de le supposer. Nous dcvons à la vérité de dire qu'elles nous ont été facilitées par Sichcl pèrc, de regrettable mémoire, dans la richc bibliothèque ophthal mologirue duquel nous avons pu compulser des ouvrages que nous avions vainement cherchés ailleurs. Il résullc de ces indications que la régénération du cristallin a été admise par tous les auteurs qui s'en sont occupés sérieusement, surtout par les auteurs allemands qu'on est certain de rencontrer dès qu'il s'agit de résoudre une question spéciale, et quc, d'une manière généralc, on a malheur'eusement ignorée ou méconnue en France. Tels sont : MM. Cocteau et Lcroy (d'Étiolles), qui ont publié Icur travail en 1825, Backlıausen, Loewenhardt et Iavidson, en 1827, Day, en 1828, Mayer ct Midlemore, en 1832, et en dernier licu Textor et Valentin, cn 1842. Tous ces auteurs, sauf Backhauscn, ont admis la régénération du cristallin, el surtout Vaicntin, le seul qui ail cxaminé au microscope des cristallins régénérés. Quant i Backhauscn, qui n'a poussé ses expéricnces que jusqu’au vingl-cinquième jour, son opinion, contraire à la régénération du cristallin, ne peut avoir de valeur scientifique.

Nalgré l'unanimité avec laguellc se sont prononcés tous les savants mentionnés, réputés par nous ì juste titrc comme des qutorités dans la question qui nous préoccupc, beaucoup d'ana. tomistes, de physiologistes et d'ophthalmologistes, ces derniers

(1) Voj. Ch. Fobin, article BHASTẺME du Dictionnaire encyclopedique des sciencos máliculcz. Paris, 1869. - Voycz aussi Julius Arnold, Dic Vorgïnge bci der Regeneralion epithelialer Gebildc. (In Archiv für palhologische Analomic u. Physiologi: u. f. Klinische Medicin. Horausg. von Rud. Virchne. 16 Bd. IIcf, II, 1. $168-$ 208. 1869 . 
surtout, contestent aujourd'luui la régénération du cristallin; et l'opéralion de l'extraction du eristallin cataracté avec sa capsule, proposée clans ces derruicrs temps, suffirait à elle seule pour démontrer le peu de cas que font certains ophthalmolonistes contemporains de la régénération du cristallin, en supposaut mème qu’ils l'admettent en principe chez les animaux.

Il est à remarquer que les cataraetotomistes ne se sont pas encore occupés d'une manière sérieuse de la section enpsulaire. Feu A. de Graefe, en introduisant dans l'art cles opérations d'yeux l'extraction linéaire modifiée, a doté la science d'une étude aussi neuve qu'approfondie sur l'incision cornéenne, et il est à regretter que cette belle intelligenee n'en ait pas fait autant pour la section capsulaire.

Dans une monographie que nous nous proposons de publier ultérieurement sur la strueture normale du cristaliin, nous exposerons la nomenclature que nous croyons la plus rationnelle de l'appareil cristallinien. Qu'il nous suffise aujourd'hui de dire que nous avons laissé aux deux moitiés de la capsule cristallinienne la dénomination de cristalloïdes antérieure et postérieure, au diamétre antéro-postérieur, passant par le centre de l'appareil cristallinien, le nom d'axe, et à ses extrémités celui de pôles antérieur et postérieur; à la partie de la circonférence du même appareil, le nom d'équateur"; el en dernier lieu la dénomination de diamètres vertical et horizontal aux droites qui passent par le centre de l'appareil cristallinien, et dont l'un a la direction du fil à plomb, et l'autre est perpendiculaire à celui-ci (pl. I, fig. 3, $\Lambda, B$.$) . Nous avons conservé le nom de globules de Morgagni ì des$ gouttelettes claires, transparentes, sans noyaux, de volumes trèsdivers, el que l'on r'egarde généralennent comme un produil post mortem des éléments du cristallin, de tubes cristalliniens aux tubes pourvus de noyaux, el de fibres cristalliniennes à ces mèmes tubes ayant perdu leurs noyaux. Nous n'avous pas cru devoir eorrserver le nom de cellules de Morgingini à des cellules que nous avions prises jusqu'ici pour des cellules épithéliales modifiécs (pl. II, fig. 2, c, et pl. III, fig. $3, c$ ), et sur lesquelles notre opinion n'est pas encore fixée Nous les avons appelées cellules de 
Robin qui, les ayant le premier distinguées des globules de Morgagni, les a à tort appelées cellules de Morgagni ou de la couche gommeuse de Morgagni, ce dernier n'ayant vu que les altérations eadavériques de eette eouche.

Nous avons fait représenter à la fin de notre travail les dessins de deux instruments et de eelles des préparations maero- et microseopiques qui nous ont paru représenter les cas les mieux réussis ou les plus intéressants de nos expériences; mais nous devons prévenir le leeteur qu'ils ne représentent pas tous exactement la strueture mieroscopique du eristallin régénéré : nous arons dessiné nos préparations telles qu'elles se présentaient sous l'objeetif du microscope, après l'emploi de certains réaetifs miero-ehimiques. Ces dessins sont, en quelque sorte, la elé de notre travail, et nous prions le leeteur de vouloir hien les eonsulter avant d'entreprendre la lecture de la seconde partie de notre munographie.

Lorsqu'il s'est agi, dans notre travail, de déerire les phénomènes inflammatoires des parties environnant l'appareil eristallinien et la régénération des éléments du eristallin, nous avons évité avee soin d'aller au fond de ees deux questions que nous reprendrons plus tard; nous avons préféré, jusqu'à nouvel ordre, lourner la difficulté et employer dans notre deseription des appellations générales, telles que a produit inflammatoire 》 et «alignement de cellules formatriees par séries linéaires. „ Ces appellalions ne préjugent rien, et nous pernettent dès aujourd'hui de montrer que nous ne voulons plus etre dupes des vanités de eertaines écoles qui s'imaginent avoir tranché ees questions, pouvoir impunément mettre de eòté les données aneiennes de pathologie, éliminer la notion des blastèmes et nier l'intervention de ces dernières dans la génération des éléments analomiques.

Les ouvrages que nous n'avons pu nous procurer et que nous avons eités d’après d'autres auteurs, sont llésignés par un astérisque.

Nous avons cru utile de mettre a la fin de notre travial une liste des auteurs qui se sont occupés de la régénéralion du crisfillin che\% l'homme of les animaux, ef les titres de leurs ou- 
vrages. Nous y avons ajouté les noms de ceux des auteurs d'anatomie, de physiologie et d'ophthalmologie, dont cetle reproduction a attiré l'attention, et qui se sont livrés à quelques appréciations, plus ou moins judicieuses à son égard, soit pour soutenir une idée préconçue (Haller et autres), soit pour réfuter celle de leurs adversaires. Mais le lecteur comprendra que nous n'avons pu citer tous les auteurs modernes d'ophthalmologie dont il trouvera les noms dans les traités de maladies d'yeux de Weeker, de Galezowski et d'autres, qu'il fera bien de consulter.

Nous nous faisons un devoir, en terminant ces préliminaires, d'exprimer notre profonde reconnaissance i nos maitres : M. W. Karavaïew, professeur à la Faculté de médecine de Kiew, à M. C. Robin, professeur à la Faculté de médecine de Paris, à M. Raynal, professeur à l'école vétérinaire d'Alforl, et à feu Sichel père, pour leur assistance et les conseils bienveillants qu'ils nous ont donnés, et dont nous avons tâché de profiter dans la mesure de nos forces el de nos moyens.

\section{I}

INDICATIONS DES EXPÉRIENCES FAITES IUSQU'A CE JOUR SLR LA RÉGÉNÉRATION DU CRISTALLIN.

Au témoignage de Mayer (1), Vrolik, eité par Buchuer en 1801 (2), fut le premier qui observa la régénération du cristallin après l'abássement de la cataracte. Plus tard, en 1S24, Dieterich reprit cette mème question, mais il était réservé à Coeteau et Leroy (d’Étiolles) d'entreprendre des expériences sur des animaux et de constater la possibilité cle la régénération du cristallin. Dans un mémoire que Leroy (d'Étiolles) lut à l'Académie de chirurgie le 10 février 1825 (3), il exposa les résultats des expé-

(1) Journal der Chirurgio u. Heillunde v. Graole u. Walher. Bd. XVIII, 1832. IV Heft. p. 548-550.

(2) * Isuchner, Waarneming van cone enlbinding der crystalvoglen, etc. Amsterdam, 1801.

(3) Leroy (d'Etiolles), Expérienees relatives à la reproduction du cristallin. Mćmoire lu à l'Académie de chirurgie, 1825. Voy. Journal de physiologie experimen- 
CHFZ QUELQUES MAMHFÈRES.

riences qu'il avait entreprises avec Cocteau sur quatre lippins, un chat ct un chien. Le procédé d'cxtraction go'cmployèrent ces savants était la liératotomic supérieure. Dans la première expérience, faite lc 27 juillet 1824 , le cristallin fut cxtrait de l'œil droit d'un lapiu d'environ trois mois. L'iris fit saillie, ct il fut impossible de le réduïre: Le mème accident se produisit au moment de l'extraction du cristallin de l'ail gauche, où, l'ir'is étant lésé, jl y eut une légère hémorrhagic. La sortie du cristallin de l'œil gauche fut hâtée au moycn d'une curette. Les accidents consécutifs ne présentèrent pas dc gravité sur l'œil gaucbe, mais il en fut autrement avec l'œil droit où une inflammation intense se déclara. L'animal fut tué le 9 août de la mème année et, à la dissection de l' ceil droit, les expérimentateurs constatèrent les lésions produites par l'inflammation générale de l'œil : la confusion inextricable de l'iris, du corps ciliaire, de la capsule cristallinienne et de la parlie antérieure de l'byalloïde. Quant à l'œil ganche, l'incision de sa membrane cristallinicnne donna issue à un cristallin lenticulaire, dont la consistance, un peu plus grande au centre qu'à la circonférence, était moindre que dans l'état naturel et se rapprochait de celle des portions excentriques du cristallin primitif. Dans leur deuxième expérience, l'opération fut pratiquée le 2 ’’ aốt 1824, sur les deux yeux d'un lapin de trois à quatre mois. L'incision de la cornée fut agrandie avec des ciseaux fins dont les pointes servirent en mème temps à diviser la cristalloïde antérieure. Le cristallin fut extrait au moyen d'une curette, et l'iris fit hernic. L'inflammation conséeutive fut intense, mais le 25 septembre, jour où l'animal fut tué au moyen d'une injection d'huilc el d'air' dans la vcine jugulaire, la cornée de l'œil gauche redevint transparente ol cellc de l'oeil droit présenta de l'opacité dans sa partic supéricurc. L'ccil gauche offrit, à l'autopsie, une membrane cristallinienne parfaitement transparente, libre d'adhérence accidentelle et contenant un cristallin lenticulaire d'une cronsistance moindre que dans l'état naturel. L'wil droit

Lale et pathologique de Magendie, 1827, t. VII, p. 30 et suivantes, et Recueil de lettres et mérnoires adressés il l'Acalérnie des sciences pendant les années $1842-$ 1813. Paris, 1814. 
ne contenait pas de cristallin. Dans leur troisième expérience, l'cxtraction fut pratiquée le 11 octobre, sur les deux yeux d'un lapin de 4 à 5 mois. L'iris fit peu hernie el l'inflammation consécutive ne fut pas intense à l'œil gauche, mais clle fut vive à l'œil droit. L'animal fut tué le 19 novembre, en injectant de l'huile dans la veine jugulairc. La dissection de l'œil gauche montra que le contenu de la capsule cristallinienne, parfaitement trensparente et sans cicatrice, n'avait pas la forme lenticulaire et la solidité du cristallin. Il était plus consistant que l'humcur vitréc et disposé en grumeaux. Quant à l'œil droit " la cavité du cristallin n'existait pas. „ Dans leur quatrième expérience, le cristallin fut extrail, lc 5 novembre $\mathbf{1} 24$, des deux yeux d'un chat adulte. $11 \mathrm{y}$ eut blessure, hémorrhagie et hernie des deux iris. L'hémorrhagie fut moins abondante sur l'œil gauche. L'animal fut tué le 6 décembre, au moyen de la suspension, et à l'examen de l'œil droit les expérimentateurs constatèrent, entre l'humeur aqueuse et l'humeur vitrée, la présence d'une cloison formée par l'iris, le corps ciliaire et la capsule cristallinienne, et dans laquelle il était impossible de distinguer quoi que ce füt, si ce n'est qu'il n'y avait point de régénérescence du cristallin. Dans l'œeil gauche ils trouvèrent un corps à peu près lenticulaire, de couleur légèrement ambrée, épais d'une demi-ligne, et analogue à une membrane épaisse ou ci une portion du cristallin desséché. L'humeur vitrée n'y existait pas. Dans leur cinquième expérience, le cristallin fut extrait, le 16 décernbre 1824, des deux yeux d'un chien de moyenne taille, âgé de quatre à cinq ans. A l'œil gauche, il y cut hémorrhagie assez abondante. L'animal périt le 31 janvier 1825 , et à la dissection de l'œil droit, faite par Gerdy, on trouva dans l'épaisseur de la cloison transparente qui séparait l'humenr vitrée, un cristallin parfaitement transparent, lenticulaire, d'une ćpaisseur et d'une consistance moindres que cclles d'un cristallin primitif. A l'œil gauche, l'iris, la capsule cristallinienne, le corps ciliaire et l'hyaloïdc étaient confondus en une sculc membrane. Enfin dans lcur sixième expérience, l'extraction du cristallin lut pratiquée, le 6 juin 1825, sur les yeux de deux lapins, dont l'un périt sans que l'examen de ses yeux pùt etre fait. Le second fut tué le 18 
CHE\% QUELQUES UAMMIFÈRES.

nocembre de la mème annéc. A la dissection de ses yeux, faite en présence de Fodera, les expérimentateurs trouvèrent que les capsules cristulliniennes étaient parfaitement transparentes et contenaient des cristallins aussi voluminenx et aussi consistants que cenx qu'ils avaient extraits. Plongés dans l'eau bouillante, ces cristallins devinrent opayues, durs et friables, absolument romme des cristallins primitifs; seulement la disposition en lamelles brillantes n'était évidente que dans les couches excentriques.

Lorsqu'on analyse chacune de ces expériences séparément, on voit que : lans la première expérience, dont la durće fut de 13 jours, le résultat a été négatif à l'œil droit et positif à l'œil ganche; dans la seconde expérience, dont la durée fut de 33 jours, le résultat a été negaitif à l' œil droit, positif à l'œil gauche; dans la troisième expérience, dont la durée fut de 39 jours, le résul tat fut négatif aux deux yeux; dins la quatrième expérience, lont la durée fut de 31 jours, le résultat fut négatif aux deux yeux; dans la cinquième expérience, dont la durée fut de 46 jours, le résultat fut positif à l'œil droit, négatif à l'œil gauche; enfin, dans la sixième expérience, dont la durée ful de 165 jours, c'est-ì-dire presque une demi-année, le résultal fui positif aux deux yeux. Ainsi dans ces 12 cas la régénération du cristallin a eu licu 5 fois.

Il est i regretter que Cocteau et Leroy (d'Étiolles), qui les premiers se sont occupés de rechercher, par voie expérimentale, les modifications (qu'apporte dans l'appareil cristallinien l'extraction du cristallin normal, n'aient pu faire usage des anesthésiques dont nous disposons aujourd'hui. Ces derniers leur auraient évilé les difficultés qu'ils éprouvèrent pendant l'opération de l'extraction et qui ne restèrent pas sans influence sur les résultats de leurs expérienccs. Quoi qu’il en soit, Lcroy (d'Étiolles) conclut, dans son mémoire, à la régénération du cristallin et alla mème jusqu'à la comparer à celle de l'os.

Les expériences de Cocteau et de Leroy (d'Étiolles) attirèrent l'attention de quelrues médccins de leur époquc, qui les répétèrent, mais qui, jusqu'i Valentin, fante d'employer l'investigation microscopirgue, firent peu marcher en avant la question de la régénération du cristallin. 
Backhausen répéla en 1827 les expériences de Cocteau et de Leroy (d'Étiolles), et en fitl'objel de sa thèse inaugurale (1). Guidé par Rudolplii cl Sehlemm, il entreprit a Berlin une série d'extractions de cristallins normaux sur huit lapins, dont 5 étaient âgés de quatre mois à leux ans el $\mathbf{3}$ autres de quatorze jours. La mélhode opératoire employée par Baekhausen était, paraît-il, la kératotomie supérieure au moyen du couteau de Beer. Pour confectionner le lambeau eornéen, il employait aussi le couleau de Siegresti. L'opération réussissait cependant rarement, à cause de l'impossibilité dans laquelle Backhausen se trouvait d'immobiliser le globe oeulaire. Des 15 eas d'extraetion qu'il fit, onze $(1,2,3,5,9,10,11,12,13,14$ et 15) donnèrent un résultat négatif, et un seulement, le $7^{\circ}$, eut un résultal positif, bien que l'auteur ail eru devoir envisager ee dernier eomme négalif; quant aux $4^{\circ}, 6^{e}$ et $S^{\circ}$ eas d'extraetion, nous ne les avons pas envisagés avee l'auteur eomme négatifs, par la raison qu'il n'y a trouvé, à li dissection des globes oeulaires, ni eristallin, ni capsule cristallinienne sans laquelle la régénération du cristallin est impossible. Sans entrer dans une analyse plus détaillée du travail de Baekhausen, nous dirons que nous n'avons pu en faire une déduetion seientifique, par la raison qu'il tuait ses lapins trop tôt et ne donnait pas assez de temps à la eapsule cristallinienne pour qu'elle pût reproduire le eristallin. En effet, la plus grande durée de ses expérienees a élé de 17 à 25 jours. Backhausen se crut cependant autorisé à nier los résultats de Cocteau et de Leroy (d'Étiolles), el fut le premier qui émit l'idée que les eristallins régénérés de ees auteur's n'élaient autre chose que les resles des eristallins laissés dans les capsules cristalliniennes, au moment de l'opération (2).

La neême annéc le doeteur Löwenhardt de Prenzlau, assisté de son eollégue Davidson, entreprit des expériences très-curieuses sur cinq lapins qu'il opéra à deux reprises pour voir si la capsule cristallinienne pouvait régénérer le cristallin, après l'extraetion

(1) De regeneralione lentis crystalline, auctor Pelrus Backhausen. Berolini, 1827.

(2) Backhausen, loc, cil., p. 20. 
d'un cristallin déjà régénćré une fois (1). Dans les dcux premiers cas il fit l'extraction des cristallins des yeux d'un lapin de deux mois ct demi par la kératotomic supéricure, ct 286 jours après (9 mois) il répéla la mème opération sur les yeux du même lapin et fit l'extraction de dcux cristallins d'une consistance moindre que celle du cristallin normal ct plus aplatis que celui-ci. Chez le second lapin, de trois mois, opéré en mème temps que le premier et par la mème méthode, il obtint le mème résultat. Li dissection des yeux, faite 88 jour's après la seconde extraction, démontra l'existence, dans les capsules, d'une petite quantité de mucus gélatineux, lequel, suivant Löwenhardt, pouvait avec le temps devenir plus dense. Chez les deux lapins suivants, l'opération secondaire fut faite 257 jours après l'opération primaire el douna lieu à l'extraction de trois cristallins régénérés, moins denses et plus aplatis que chez les deux premiers lapins; de l'un des yeux du dernier lapin non-seulement il ne sortit pas dc cristallin régénéré, mais à sa dissection on n'en trouva pas de trace. Il faut dire que l'inflammation de cet œil après l'opération fut très-intense. Enfin chez le cinquième lapin, au moment de l'opération primaire, l'opérateur non seulement détruisit la cristalloïde antérieure, mais dilacéra dans plusieurs directions la cristalloïde postéricure; l'opération secondaire, faite 264 jours après, ne donna issuc qu'à une petite quantité d'humeur vitrée. De toutes ces expériences Löwenhardt conclut: que le cristallin se régénére chez les animaux, que chez les jeunes animaux cette régénération s'opéré plus vite, et que la capsule cristallinienne est l'urgane régénérateur du nouveau cristallin. Bien que ces résultats ne préseutassenl pas un intéret pratique, il n'en est pas moins vrai que les expériences sur lesquelles ils reposaient élaient intéressantcs el instructives : clles démontraient la possibilité d'extraire les cristallins reproduils ct par conséquent n'allhérant poiut, ou du moins faiblement, is la capsule cristallinienne; clles démontraient également la forcc régénératricc très-faible de la capsule

(1) D). Liwenhardt aus Prenzlau, Einigo Versuche, um die Regeneralion der Kryslallinse su documentiren, in Neue Nolizen u. s. w. von Froriep. No 418 (n०22 des XIXtu bandes), Sejlember, 18/1, Spalte 344-340. 
cristailinienne, après l'opération primaire, et la nécessité de sì présence pour la reproduction du cristallin extrait.

En 1528, Henri Day (1) relata dans la Gazette médicale "The Lancet „ l'cxpérience qu'il fit sur un lapin, à l'œil duquel il fit la liératotomie et l'extraction da cristallin normal ; 43 jours aprés l'opération it tua l'animal ct trouva dans l'œil opéré un eristallin régénéré de forme ordinaire, moins dense que le cristallin normal.

En 1832, Midlmore inséra dans The London medical Gazetle (2) les résultats dcs expérienccs qu'il entreprit, dans le bul de vćrificr celles de Cocteau et de Leroy (d'Étiollcs). Il fit l'extraction du cristallin : $1^{\circ}$ de l'œil d'un lapin d'environ trois mois, et, en disséquant eet œil 9 jours après, il n'y trouva pas de trace d'un cristallin régénéré; $2^{\circ}$ de l'œil d'un lapin d'environ 4 mois, et 63 jours après il y trouva, à la place du cristallin, une substance demi-gélatincuse entourće d'une autre plus consistante et parfaitement transparente; enfin $3^{\circ}$ de l'œil d'un lapin d'environ 5 mois, et en dissćquant cet œil 61 jour's après, il y trouva un cristallin régćnéré lequel, par sa forme cl sa structure, ressemblait parfaitement à celui qu'il avait extrait. A la périphérie de ce cristallin régénéré, il trouva une petite cavité remplie d'un liquide dont la densité était entre celle de l'humeur aqueuse et de l'humcur vitreuse, et différait, du reste, du cristallin tout i fait normal. De ces trois expéricnces, dont le résultat a été négatif dans lc premier cas el positif dans les deux autres, et de plusieurs autres qu'il ne fit que mentionner, Midlmore conclua : $1^{\circ}$ que la régénéwration du cristallin a lieu chez les jeunes animaux lorsque les deux cristallö̈des n'adhèrent pas l'une à l'autre après l'opération, quand la cristalloïde postérieure n'est pas lésée el, en dernier lieu, lorsque l'inflammatiou du globe oculaire n'est pas intense ; $2^{\circ}$ que le cristallin régénéré est liquide d'abord et acquiert sa forme avant d'arriver au degré voulu de densité normale ; et cnfin $3^{\circ}$ que le cristallin reproduit ne peut acquérir son entier dévcloppement qu'un an apprès l'extraction du cristallin normal, et que ce

(1) H. Day, Reproduction of the iens (The Lanccl. 1828, 15 nov., p. 212).

(2) Midlmore, loc. cil., p. 344 et 345 . 
diveloppement dépend do l'âge des animaux pris pour les cxpériences, ainsi que de plusieurs autres circonslances.

.llillmore partigeait les opinions de ses contemporains sur la structure du cristallin, bien qu'elles fussent basées sur les travaux des anatomistes du siècle passć, tcls que : Winslow, Albinus, IIovius cl surtout Zinn, qui donna, dans sa description de l'œil humain (1), le dessin des vaisseaux de la capsule cristallinienne. Cela notis expliquc pourquoi il attribua une grande valcur à la cristalloïde postćrieure et élablit une corrélation intime cntre sa Ićsion et la régénération du cristallin. Cela nous explique aussi pourquoi il admettait la régćnćration dn cristallin chez les jeunes sujets dont la cristalloïde postérieure n'avait pas été lćsée pendant l'opération; les vaisseaux de cette cristalloïde, selon Midlmore, n’étaient pas en état, chez les vieux animaux, de coopérer à la régénération du cristallin (2). Midlmore admcttait en outre que le cristallin peut se régénérer deux, el même trois fois, après l'extraction répćtée des cristallins (3).

La mème annéc Mayer, professeur à Bonn, publiail dans le journal de Graefe et de Walther le résultat de ses expćriences sur la régénération du cristallin (4). Il fit l'extraction clu cristallin dc l'ocil gauche de 14 lapins, par le procédć de la kératotomie. Chaque jour un des cinq premicrs lapins fut lué pendant les cinq premicrs jours, mais à la dissection de leurs yeux l'auteur nc trouva pas de cristallin régénéré. Dans l'œil dn dernicr lapin, il trouva la capsule cristallinienne lacérće el ayant un trou à son centre. A l'cxamen de l'oil du $6^{\mathrm{c}}$ lapin, tuć le $6^{\mathrm{e}}$ jour après l'extraction du cristallin, il frouva à la place de ce dernicl un petit anneau, rempli de substance cristallinienne molle et se détachant facilement de la capsule cristallinionne; dans le globe oculaire du $7^{\circ}$

(1) Descriptio analomica oculi humuni iconibus illusrala, auclore Johanne Grollfried Zinn. Gollinges. 1775, p. 138-142.

(2) On the reproduction of the cristallinc lcns, by Midlmore (Tho London medical Gazelle. 1832, vol. X, June. 16, P. 341-348).

(3) Idern., p. 3.18

(1) Ueber dic leproluclion der Kristallinse von prof. Mayer in Bonn (Journal der Chirurgie und Augenhcilkunde v. C. R. v. Gracfe und Ph. Wallhcr, 1832, bir. Yvil, lleft. 4, p. 521 et suivantes). 
lapin, tuć 28 jours après l'opération, il trouva í la place du criscallin extrail un anneau assez épais. A la dissection de l'œil du $\delta^{e}$ lapin, lué 52 jours après l'opération, le cristallin régénéré était presque aussi grand que celui qui avait été extrait; mais il adlı́rait à la capsule el avait unc lorne annulaire, avec un trou au milicu. L'œil dı $9^{\mathrm{e}}$ lapin, tué 56 jours après l'opération, présentait, à la place du cristallin extrait, des grumeaux réunis et formant un anncau; dans les ycux du $10^{\circ}$ lapin, lué 56 jours, et des $11^{\mathrm{c}}$ et $12^{\circ}$ lapins, tués 49 jours après l'extraction du cristallin, Hajer trouva, ainsi qu'il s'exprime, "de beaux anneaux épais „, avce un lrou au milieu; ct il ajoute qu'il montra 1 cristallin du $10^{\circ}$ lapiu à Walther, Tiedemann, Treviranus el $\mathbf{F}$ tué 120 jour's apprès l'opéra'

1). Daus l'œil du $13^{c}$ lapin, a les mèmes grumeaus réunis en anncau qu'il avait trouvés chez le $9^{\circ}$ lapin; cnfin dans l'œil du lapin, lué 132 jours après l'extraction du cristallin, il trouva m cristallin régénéré, dont la circonférence était plus grande que celle du cristallin cxtrait. Ce cristallin avait à son milieu un trou qui correspondait à la lésion de la capsule cristallinicnne faitc pendant l'opération.

Maycr, en se basanl sur scs cinq cxpériences avec résultal positif et neuf expériences avec résullat négalif, arriva aux conclusions suivantes : $1^{\circ}$ le cristallin, après l'extraction du cristallin normal de l'œil, se régénère; toutefois il faul que sa capsule soit saine; $2^{\circ}$ la régénération du cristallin dépend de la cristalloïde antérieure; $3^{\circ}$ le cristallin commence à se former d'abord à la circonférence de la capsule cristallinienne, puis alleint jusqu’au milicu de cette même eapsule à l'endroit où cette dernière a été lćsée pendant l'opération, et s'y arrete dans sa régénération; $4^{\circ}$ la capsule cristallinienne est toujours plus ou moins adliérente au cristallin régénéré; $5^{\circ}$ la régénération du cristallin régénéré et extrait de l'œil n'a plus lieu; $6^{\circ}$ le cristallin régćnéré a la même transparence et la mêne densité que le cristallin normal extrait, el peut au point de vuc plyysiologique le remplacer plus ou moins; $7^{\circ}$ enfin, le cristallin régénéré cst toujours plus pelit que le cristal-

(1) Mayer, l. ci, p. 535. 
lin normal. Ces experriences, qui dénotirent chez l'autcur en question une grande finesse d'observation el $n$ lalent incontcstable d'expérimentation, furent très-importantcs : elles lui fireut eonstater que la régénération du eristallin dépend principalement de la partie équatoriale de la eapsule cristallinienne. Pour Mayer, lui-même, ees expériences avaient cneore une autre importance : elles confirmaient ses idées théoriques, d'après lesquelles il rapportait le cristallin aux tissus feuilletés (Bläter-Gewebe) (1), et qui étaient doués, selon lui, d'une grande force reproductive (2).

D'après Chelius (3), le docteur Pauli de Landau (4) fit, cn 1838, l'extraction du eristallin les yeux d'un vieux ehien et d'un bouf. Il examina les yeux du premier 163 jours après l'opération, et ne eonstata pas sur l'un de ees yeux la présence d'un cristallin régénéré, mais en trouva un dans le second. Le nouveau cristallin était moins dense que eelui qui avait été extrait. Dans les yeux du bouf, tué 211 jours après l'opération, il trouva des cristallins régénérés; ceux-ci étaient moitié moins grands que le cristallin nurmal et moins denses que le cristallin régénéré du chien.

En 1842, parut le travail de Textor (5) sur la régćnération du eristallin. Textor s'adonna particulièrement à l'étude des yeux humains soumis à l'opération de l'extraction de la cataracle, et s'il fit quelques extraetions du eristallin normal sur des animaux ce ne fut que pour affermir, en quelque sorte, l'opinion qu'il s'était faite sur la régénération du cristallin et pour vérifier les expériences de Cocleau et de Leroy (d'Etiolles). Il fit l'extraetion du erisiallin uormal, au moyen de la kératotomic, des deux yeux

(1) "Nayer, Leber Hislologie und eine neue Eintheilung der Gewebe des menschlichen Korpers. Bonn, 1819, p. 14.

'2, Nayer, Ueber die Reprod., etc., p. 521.

(3) Max. Jos. Chelius, Ilandbueh der Augenhoillinde, 2 Bd. Stultgart, 1839, p. 331-332. - Traité protique d'ophthaimologie, par Max. Chelius. Trad, par Ruef ế Deiber, 1839, p. 314 .

(1), "Mauli, Veber den grauen Staar u. die Verkrilmmungen. Slultgart, 1838.

(5)) Veber die Wiedererzougung der Kryslallinse. Inaugural-Abhandlung $v$. karl Texlor aus Mïnchen, mil 3 Sleindruclilnfeln. Wiirzburg, 1842. 
de deux lapins de quelques semaines el de deux autres lapins adultes. L'oil droit du premier lapin, frar suite d'une inflammation intense, suppura et s'atrophia ; son wil ganche ne subit ni inflammation; ni suppuration, mais, à la dissection, faite 231 jour's après l'opération, Textor n'y trouva p'as de cristallin régénéré. A l'oeil droit du second lapin il se fit une atrophie par les mèmes raisons que chez le prenier lapin; à- l'œil ganche qui daus l'ouvrage de l'auteur cst appelé, par erreur', wil droit, T'extor trouva, 232 jours après l'opération, un cristallin régénéré transparent et gélatiniforme; il se présentait sous formic s'anncau épais de bas en haut et mince dans la direction du diamètre horizontal. Il y avait au milieu de ce bourrelet une fente transversale fermée par une membrane très-délicate. Dans les yeux du $3^{\circ}$ lapin, mis à mort ça jours après l'opération, l'auteur ne trouva pas de eristallins régénérés. Dans les yeux du $4^{\circ}$ lapin, tue 212 jours (7 mois) après l'extraction du cristallin qui, à la suite d'une contraclion spasmodique des muscles de l'œil, sortit seul des globes oculaires, Tex tor trouva dans les capsules cristalliniennes transparentes un peu de mucus gélatiniforme qu'il supposa etre des cristallins régénérés. Ainsi, sur huit cas d'extraction du cristallin normal chez des lap̣ins, le cristallin ne se régénéra que trois fois, ce qui n'empêcha pas Textor, non seulement d'admettre la régénération du cristallin, mais encore de formuler les conclusions suivantes (1) :

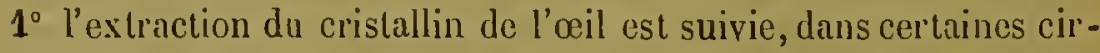
constances, soit de la régénération d'un cristallin plus ou moins normal, soit de la formation d'une pelite quantité de la substance cristallinienne; $2^{\circ}$ la régénérntion du cristallin dépend de la capsule cristallinienne (matrix); $3^{\circ}$ après l'extraction de la capsule cristallinienne de l'ocil, le cristallin ne se régénère pas; $4^{\circ}$ le bourrelet cristallinien est tonjours adhérent à la capsule; cette adhérence u'est cependant pas assez forte pour que l'on ne puisse l'en extraire; $5^{\circ}$ le cristallin régénéré est aussi transparent que le eristallin normal et l'esprit-de-vin excrce sur tous deux la mème action; $6^{\circ}$ la régénération du cristallin, pour avoir

(1) Karl T'exlor, l, c., p. 46. 
lieu, exige un certain temps; $7^{\circ}$ la densité, l'épaisseur et la quanlité de la substance cristallinienne régénérée augmentent à mesure qu'on laisse s'éloigner le moment de la dissection de l'œil opéré de celui oú a eu lieu l'opération; $8^{\circ}$ la forme du cristallin régénéré dépend de la lésion de la capsule cristallinienne et de la manièrc dont celte dernière se cicatrise : si c'est au milieu qu'elle se cicatrise, il se forme un bourrelet eristallinien; si cette cicatrisation a lieu ì un endroit quelconque de sa partic équatoriale, le cristallin régénéré prend la forme demi-lunaire; $9^{\circ}$ dans tous les cas de régénération du eristallin, la capsule cristallinienne reste transparente.

A ces condusions Textor en joint dans son travail encore trois que nous passons sous silence, vu qu'elles ne se rapportent qu'aux cristallins humains régénérés.

L'ouvrage de Texior' est précieux par le déve!oppement que son auteur y doma à la partie historique; il occupe dans l'histoire de la régénération du cristallin une place non moins remarquable que celle qu'occupe le travail de IV. Sœmmering, intitulé : "Observalions sur les changements organiques dans l'œil, après l'opération de l'extraction de la cataracte " (1), bien 'ju'il n'ail eu ni le mème relentissenent, ni la mème influence sur les médecins de l'époque. On sail que W. Scommering, fils de l'illustre S. F. Sommering, présenta à son père sa monographie, le jour du cinquantième anniversaire du doclorat el de la carrière scientifirgue de ce dernier. Halheurcusement, dans l'ouvrage de Texlor, se trouve une lacune que sentait l'auteur lui-mème (2), c'est le manque de recherches nicroscopiques; el c'est i Valentin, professeur it Berne, qu'il était réservé de la combler.

Valentin fit, cu 1842, l'extraction du cristallin normai par la teratolonic inferieure des yenx de denx lapins, mais il ne mublia le resultal de ses cxpériences gu'en 18/4, dams le jommal de Henle cet l'feifer (3). En cximminant l'wil d'un jeune laprin, lue par

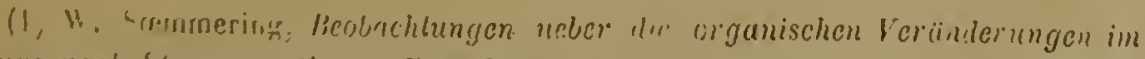
duge nark Slauroperationen. Francfurl. 1828.

(2) Karl Texlıs, l. c. Finrede. 1. vil.

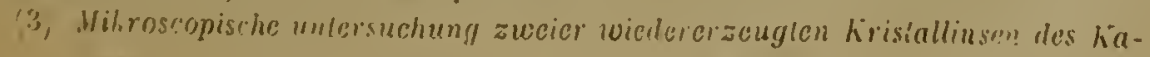

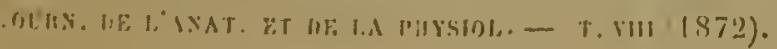


la strangulation, 116 jours après l'operation, il y trouva le cristallin régénéré transparent, de forme ronde et un peu aplati. Son diamétre était de $41 / \mathbf{L}^{\prime \prime \prime}$ (9 millim.), tandis que celui du cristallin normal ćtail de $43 / 4^{\prime \prime \prime}$ (9 1/2 millim.); mais d'avant en arrière, c'est-il-dire dans son épaisseur', il n'avait que la moitié du eristallin normal. $\Lambda$ sa partic inférieure lo cristallin régénéré présentait un espace triangulaire opaque de $2^{\prime \prime \prime}$ (4 millim.) de long. L'exploration microscopique démontra que le cristallin était composé de cellules cristalliniennes, enractéristiques, rondes el polyédriques, les plus petites de $0,006-0,010^{\prime \prime \prime}$, les plus grimdes de $0,013-0,030^{\prime \prime \prime}$, et de tubes d'un dianétre de $0,0035^{\prime \prime \prime}-$ $0,0070^{\prime \prime \prime}$. Ces derniers, ramassés en grumeaux, étaient plus délicats que les tubes normaux dont le diamètre était de 0,0025 $0,0055^{\prime \prime \prime}$. Dans l'œil du second lapin adultc, tué également 116 jours après l'opération, le même expérimentaleur trouva un cristallin régénéré de $31 / 2^{\prime \prime \prime}(7 \mathrm{l} / 4 \mathrm{~mm}$.$) , — Landis que le cristallin$ normal avait $4 \quad 1 / 2^{\prime \prime \prime}$ de diamètre $\left(\begin{array}{llll}9 & 1 / 4 & \mathrm{~mm} .\end{array}\right)$, - aplati et adhérant à la cicalrice de la cornée. Il ćtait transparent et avait à son centre el un peu au-rlessous de celui-ci un espace opraque, jaune et rempli d'une substance granuleuse. L'investigation microscopique y démontra l'existence des mèmes éléments que dans le cristallin régénéré dlu premier lapin.

De ees expériences, Valentin conclut ì la régénération du cristallin, régénération yu'il expliquait, d'après la théorie de Sehrvann, par l'épanchement dians la capsule eristallinienne, du hlastéme (eytoblastmasse) el la naissance des cellules et des tubes. Il émit l'opinion que les parties lésées de la capsule cristallinienne ne se régénèrent pas, que la régénération du cristallin ne peut avoir lieu qu'en tant que la eapsule cristallinienne reste ì sia place, que e'est i clle qu'est due la forme du cristallin régénéré, et, en dernier lieu, qu'à la rrégénération du cristallin coopèrent les vaisseaux de l'iris el du corps cilinire.

ninchens v. Valentin (Henle u. Pfeifer, Zeilschrifl f. ration. Mcdicin. 1844, 1 Bd 2 partie). Voyez aussi C. Chr, Schmidt's Jahrbicher d. gesammlen Vodicin. Jahrg. 1814, 12 tan Bd. no2. 
Si nous ajoutons ì tous ces experinentateurs: Dicterich (1), cité par Textor (2), qui avait observé lá régénération partielle $\|^{4}$ cristallin aprés lit lésion de sa capsule, Balling et Gruby (de Vienne), cités prar ce même Texlor (3), qui firent des expériences sur des animalts et obtinrent des cristallins régánéés, Ross (4), cicé par Hyrtl (5), qui fit égalcuent des expériences sur des lapins, feu $S$ chel (6) pére, qui dans sa lemarquable "Iconograplice ophlhalmologique s parle, en passant, de ses expériences sur des lipins, mais qui conclut à la non-régeneration du cristallin, enfiu Hanmann (7); cilé par Desmares (8), qui, par contre, conclut à la régénéraion du cristallin, sans toutefois appuyer par des expćriences personnelles celte conclusion, nous aurons terminć la liste des savants qui se sont occupés d'une manièe spéciale de la régéıération du cristallin.

Depuis Valentin, personne ne s'occupa de la régénération du cristallin, et on serait porté ì croire yue ses expériences avaient été considérées comme courommenent de l'ouvre édifièe par ses prédécesseurs, el que les anatomistes, les physiologistes et lcs ophthalmologistes avaient accepté comme suffisamment démontrée la régénération du cristaliin extrait, sinon che\% l'homme, du moins chez les animaux. Il u'en fut ricu cependaut, ot nous arons déja insisté daus nos preiminares sur la différence d'opinions qui existe encore aujourd'hui parmi nus savants conlières à l'égard de cette gutestion. On peut done dire que Valentin ourrit une nouvelle voie a ceux qui, comme hous, devaient aprés lui reprendre la question de la régénération du cristallin.

(1 "Fried. Chr, Wielerich, Ueber die Verl"undungen des Linsensyslemes. Tübiu" कूen, $182 \%$.

'2) K. Textnr, Ucber die Wiedererzengung der Kryslallinse. 1842, p. 34.

(3) K. Textor, l. c., p. 34. (Substantia lontis crystallince in eane regeneralur. Gruby.)

'1, Piosz, Ilandbuch der chirurgisehen Anatomic. Annce?...

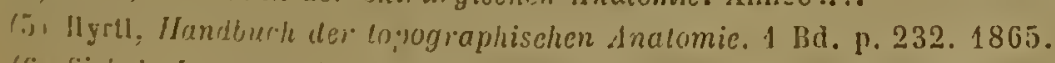

'f; Sirhel, Ieonographe uphlhalmologique. P'aris, 1852-j9, 1). 269.

7) Ilanmann, Uetser lieproduclio lentis, von docteur llanmann zu liostock. In Hufland's Journal der praklischen lleillunde. Janvicr 1842.

8) Lesmarres, Traté thrimique el pralique des malulies d'yeur. Haris, 1858. $2^{\circ}$ inl. 1. III, 1. .386i. 
En 1867, nous lìmes unc communication il l'Acadénie des scicuces (1) dans laquellc, tout en donnant une nouvelle et compléle démonstration do: la régrincration du rristallin, nons consignames les résultats de nos reclurelics microscopiques sur des cristallins régénérés de quelques animaux mammifères. In all après nous publictnes en langue russe, in extenso, le mèrne travail que corroboria cu quelque sorte, plus lard, Philipeaus, dans une commuiicalion que lit en son nom Vulpian à la Sociélé de Biologie (2). Plii lipeaux pratiqua l'extraction dı cristallin normal au moyen de la kératolomie inférieure sur six lapins àgrés de trois ans (roy. pl. 1, fig. 3 el 4). "Unde ces lapins ", dit-il dans sal communication, a a étć examiné 15 jours après l'opération : la capsule śtait tumélićc, épaissie, et elle paraissail contenir déjà mu rudinent de tissu cristallinien. Un second lapin a été examinć 25 jours aprćs l'expérierıce. La caysule étail beaucoup plus épaissie que clıez l'animal précćdent; l'épraississement siégeait surtout au voisinage du bord adhérent de la capsule. Chez le troisieme lapin, sacrifie 40 jours: après l'expérience, l'épaississement élait encorc plus prononce; lc tissu de cet épaississement, semblable à celui du cristallin, formait un anneau complet en dedans de la circonférence de la capsule. Les trois autres lapins ont été examinis : l'un alu bout de 60 jours après l'opération, un aulrc au bout de 4 mois et le derniei au bout de 5 mois el 20 jours. La régenération était de plus en plus avancée; et chez les deux dernirers clle flait complète ou à peu près : le cristallin avait reconvé sa forme lenticulairc, el, chez le dernier, it avait presque son volume normal. "

Ces expériences, dúnt les résultats, c'est-i-dire les cristallius régénérćs, furent mis sous les ycux des membres le la Socićlć de Biologie, dans la séance du 23 avril 1870, présidće par .11. Brown. Séquard, confirmèrent nos recherelies ainsi que celles de noz prodécesseurs. is fecteur comprendra facilement l’imporlance que

(1) Comples rendus de l'Accidemie des sciences. 28 janvier 1871.

(2) Philipeaux, Expériences monlrant que le crislallin peul so vegeinerer chez le's mammifères par une formation nouvelle dans lu capsule crislallinicune, in Gaselle médicale, 1870, no 46. 1). 577 (séance du 23 avril 1870 de la Société de Biologie). 


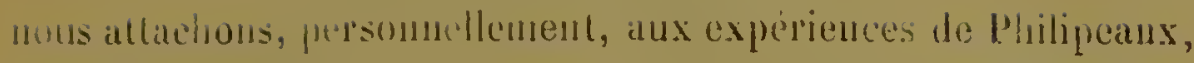
si nous lui apprenons qu'elles furent entreprises à l'insu des nòtres, ct qu'elles nous donnèrent gain de: cause contre eertains ophthalmologistes qui, parfinitement ignorants de la question qui nous occupe, n'en opposérent pas moins à notre travail une négation aussi impuissante que de mavais aloi.

\section{II}

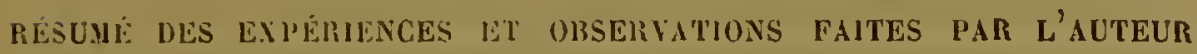
SUR LA iÁGÉNÉRATION DU CKISTALLIN.

Avant d'exposer les expériences que nous entreprimes sur des animaux pour éludier les phénoménes qui se produisent dans leur appureil cristallinien, après l'extraction du cristaliin normal, nous devons dire que nos douze premières expériences furent faites à la fin de 1862, à Kiew, oì nous eûnes l'honueųr de démontrer à la Société des médecins de Kiew, dans sa séance dia 21 septembre 1863 , deux yeux rle lapin avee des eristallins régénérés. Toutes les expériences que nous fimes à Kiew étaient entreprises sur des lapins, et ce ne fut que plus tard, à Paris, que nous pûmes, grâce aux professeurs liobin el Raynal, à ce dernier surtout, qui mit à notre disposition des moutons, des brebis et des chiens, les reprendre sur une grande échelle. Nous avons fait l'exiraction du cristallin normal, par la kératotomie, 19 fois chez. des lapins, 17 fois chez des chiens, 2 fois chez des moutons, 2 fois chez des brehis, 4 fois chez des chals, 3 fois chez des cochons d'Inde, 2 fois chez des rals, en tout 49 fois. Sur ces trois derniers aninaux, ainsi que sur quelques lapins, nous avons fait nos expériences daris le laboraloire d'listologie de la faculté de médecine dre l'aris.

Afin d'extraire plus facilenent le cristallin, nous chloroformions les animaux apres avoir préalablement introduit dans leurs veux quelques goultes d'une solution d'atropine, el ce n'est que dans les cas ois les animaux du laboratoire appartenaient it d'autres sxpérimentatemr, et oil nous opérions inopiuément, ou bien dant cenx nil liris, ansiğque nous avons pul le constaler 
chez les chieus, se dilatail suffisamment sous l'inlluence du chloroforme, que nous n'employânes ni ce clernicr, ni l'atropine.

Le procédé opératoire que nous mmployâmes dans nos expériences était le suivent : après aroir dilaté l'iris, nous chloroformions l'animal; nous incisions ensuile l'angle externe de l'xil chez ceux des animaux qui ayaient la fente palpébrale petite; nous fixions le globe oculaire as moyen de petites piuces (de Blömmer), et portant la lame du kératotome de Beer ou de eclui de Zelicuder parallélement au plan de l'iris, nous la faisions pénétrer à l'exirćmilé externe du diametre transversal de la cornéc. Quand la pointe du couteau avait traversé colle-ci, nous poussions l'instrument de dehors en dedans et nous obtenious, en contiuuant la section cornéennc, un lambeau supérieur ou inféricur, sclon que le tranchant du couteau était tourné en haut ou en bas. Afin de nous faciliter l'extraction du cristallin, unous tàchions de taire la section de la cognée le plus près possible de la conjonelive, el nous obtenions ainsi un lambeau gerand et régulier, ce qui eependant ne nous réussissait pas toujours. Dans quclques eas, avant de terminer la confection du lambeau nous linissions une petile bride étroite de cornée que nous ne coupious, soit avec de petits ciseaux, soit avec le petit coutcau mousse de Desmarres, que lorsaue nous avions fait l'incision de la capsule cristallinienne. Au début de nos expériences, uous faisions l'ouverture de lis capsule par le procéclé ordinaire, c'est-d-dire qu'après avoir terminé la section de la cornée, nous faisions, ì l'aide du lijstitome, l'incision longitudinale ou cruciale de la cristalloïle antérieure. Plus tard, ayant compiris l'importance de ce tempss d'opération, nous làchames de laire à la capsule cristallinienne une incision simple el demicirculaire, et correspondant, à peu près, à l'insertion de la 7.onule de Zinn, ce qui, théoriquement parlan!, devail nous domner un lambeau capsulaire pareil à celui de la cornée. Pour faire l'incision do la capsule cristallinienne, nous cmployâmes soit le kystitome, soit l'aiguille ì cataracte ordinaire, soil celle de Cheselden, soit une aiguille genonilléc. confectionnéc sur nos indicalions par Lïrer (pl. I, fig. 1, b), snil enfin l'aiguille de Chrselden modifiée (pl. I, fig. 1, a). Nous derons dire que nous n'oh- 
CHEZ QUELQLES MAMMGEHS.

timmes pas un grand bénéfice de l'aiguilic genouillée. Hans les cas où le cristallin, aprés l'iucision de sa capsule, ne sortait pas de lui-mème ou n'était pas expulsé spontanément (ce qui arrivait lorsque les contrictions spasmodiques des muscles de l'œil étaient fortes), nous obtenions sa sortie en pressant légèrement, au moyen de la curetle de Daviel, sur la partie du ghobe oculaire opposée à la section cornéenne.

Afin de nous faciliter l'incision demi-circulaire de la cristalloïde anlérieure de la capsule, nous faisions, dans quelques cas, l'excision d'une partie de l'iris ou bien nous dilacérions ce dernier au moyen de petits crochets mousses. Il nous est arrivé aussi quelquelois d'introduire, avant d'opérer la section de la cornée, l'aiguille à calaracte dans la chambre antérieure, de faire l'incision de la capsule, el, après avoir attendu quelque temps, de passer aux autres temps de l'opération. Afin de tenir les lévres de la plaie cornéenne le plus longtemps possible affroutées, nous réunissions les paupières par une ou deux sutures qui tombaient ordinairement entre le $3^{\mathrm{e}}$ et le $5^{\mathrm{e}}$ jour. Ces sutures tombaient plus tôt, si le résultal de l'opération était négatif, c'est-à-dire sila suppuration de l'œil se déclarait. Nous avons tenu quelques-uns de nos animaux dans des lieux obscurs; à quelques autres, dans le but de garantir leurs yeux opérés, nous fixions leurs propres oreilles, au moyen de sutures, au-dessous des orbites. Nous devons dire cependant que toutes ces précnulions ne contribuaient pas loujours à nous faire obtenir des résultats plus favorables yue cewx que nous obtenions saus elles.

Parmi les phénoménes qui se produisaient dans nos expériences après l'opération de l'exlraction ducristallin, nous devons mentionner : $1^{\circ}$ la gucirison de la plaie cornéenue, sans complication aucune, le rélablissement des fonclions de l'iris, en un mot, et, d'une maniẻe générale, l'issue firvorable de l'opération; $2^{\circ}$ l'inflammation pen intense de lis cornce de dre l'iris. Dans ce ras, l'inflammation so terminait par une résolulion pure et simple, ou bien clle ratail suivie d'adliérences (le plus souvent de filaments) de l'ii is aver la cicalrice de la plaie crincernue ou ares relle de la capsule cristallinienne, restec it a place apres l'ope 
ration de l'extraction dn cristallin el renfermant ou non un cristallin régénéré; ou bien enfin elle donnait lieu à de fausses membranes constituces par du tissu conjonctif de nouvclle formation et du pigment, et lapissant la capsule cristallinicune (cataractes siliqneuses); $3^{\circ}$ la panophthalmie el, comme conséquence, l'atrophie plus ou moins prononcée de l'œeil. Ici nous observions, à côté de la procidence de l'iris, le rnmollisscrnent de l'hımcur vitrée; l'apparition çà et là de flocons formés par des cellules modifiécs de l'humeur vitrée; l'inflainmation du corps ciliaire, de la choroïde, cle la rétine; le décollement de cette dẹnière, son ratatinement; l'absorption de l'humeur vitréc et la formation d'une espèce cle cordon, qui allait comme une chalaze, de la prapille du nerf optique à la cristalloïde postéricure el que constituaient la rétine dégénérée (apparition au milieu de ses éléments du (issu conjonclif), la membrane hyaloïde du corṇs vitré et les cellules modifićés de ce dérnier. Les cristalloüdes se retrouvaient avec difficulté au milieu des tissus modifiés et pour la plupart liés entre eux par du tissu conjonctif de nouvclle formation.

Pour examiner les changements qui s'opéraient dans l'appar'eil cristallinien, après l'extraction du cristallin normal, nous enlcvions la cornée du globe oculaire en la coupant avec de petits ciseaux tout autour du cercle extérieur de l'iris et en la laissant quelquefois attachée à la sclérotique au moyen d'une toute petile bride. Nous coupions ensuite avec ces mèmes ciseaux, an milieu du globe oculaire, la sclérotique, la choroïde, la rétine et l'humcur vitrée, et nous divisions ainsi l'oil en deux moitiés, l'une antéricure et l'autre postérieure ; cnfin nous éloignions tout à fait la cornće en coupant sa bride, et nous enlevions l'iris ct ce qui restait d'humeur vitrée du còté de la cristalloïde postéricurc.

L'examen pratiqué de la sorte sur 49 globes oculaires extirpés chez différents animaux nous montra que : sur 19 yeux de lapins le cristallin s'élait régénérć 7 fois; sur 17 yeux de chiels, 5 fois; sur 2 ycux de bélier, 2 fois; sur 4 ycux de clat, 1 fois; sur 3 ycux de cochon d'Inde, 2 lois; en tout 17 cristallins régénérés. La non-régénćration du cristallin cut lieu : 12 fois chez les lapins, 12 fois che\% les ehiens, 2 fois chez les brehis, 3 fois chez les 


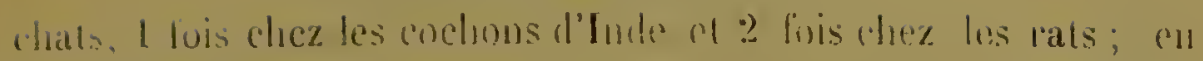
tout 32 fois.

Forme des cristallins régénérés. - Les cristallins régénérés, obtenus dans nos expériences, avaient la forme : $1^{\circ}$ du cristallin normal extrait, c'est-i-dire, ils étaient arrondis dans leur partic équatoriale, mais toujours plus ou moins aplatis de devant en arrière, r'est-ì-dire diuss la direction de leur axe (diamétre antéro-postérieur) (pl. I, fig. 3, B); $2^{\circ}$ d'un anneau formé par la partie équatoriale de la capsule cristalliniemne. Ici les bords de la cristalloüde antérieure, produits par la lésion an moment de l'opéralion, se recroquevillaient, s'éloignnient du pòle antérieur et adhéraient à la cristalloïde postérieure au moyen d'une substance imorphe; la cristalloïde postérienre formait ainsi, seule, la fossette de l'anneau cristallinien, c'est-à-dire, l'espace qui se trouvait au milieu de l'anneau constitué, dans les cas à résultat positif, par des éléments du cristallin, et dans les cas à résultat négatif, par du tissu conjonctif de nouvelle formation, du pigment, elc. (vor. pl. I, fig. 2, d) ; $3^{\circ}$ d'un fer à cheval formé par l'anneau dont les cristalloĩdes étaient adhérentes dans un endroit quelconque. La fossette annulaire se présentail ici ingrandie par suite de soll prolongement jusqu'à l'endroit interrompu de l'anneau (voj. pl. II, fig. 2, a, et pl. III, fig. 3,a); $4^{0}$ demi-lunaire par suite de ce que le cristallin régénéré formait un bourrelet qui n'occupait qu'une partie quelconque de l'équateur de la capsule cristallinieune, dont les cristalloüdes étaient, partout ailleurs, adhérentes l'une a l'autre (voy. pl. VI, fig. 1, B. $a^{\prime}$ ); enfin $5^{\circ}$ la forme irrégulière, à surface bosselée, par suite de produits inflammatoires occupant la fossette cristallinienne, ou bien la surface antérieure de la capsule, el par suite de l'apparition d'éléments cristalliniens, par endroits seulcment de la partie équatoriale de cette mème capsule.

Xinns avous examiné presque toutes les préparations du cristallin lérriné, soit plus on moins complétement, srit partiollement, immédiatement ou bientot aprís l'extirpation des yeux chez les anir aux operés. Afin de unus laciliter l'examen microscopique d. cres proparations, nous y ajoulions les solutious de 5 pour 100 
l'acide sulfurique, de 20 pour 100 d'acide acelique dilué et de 10 pour 100 de polasse causlique. Pour colorer nos préparations nous avons employé une dissolution de carninin, peu riclıe en ammoniaque; pour les durcir nous arons employé une solution de bichromale de polassium, 3 parties pour 100 d'eau, el enfin pour les conserver nous avons employé de la glycérine pure ou bien un mélange en parties égales: de gylycćrine, d'une solution concentrée d'ichthyocolle et d'une solution saturée d'acide arsénicux, auxquelles nous ajoutions un peu de camphre (mélange de Legros).

Avant de décrirc la structurc intime du cristallin régénéré, nous croyons utile d'entrer dans quelques explications sur ce que nous enlendons par régénération du cristallin, et, lorsque celle·ci a lieu, quelles sont les phases de son évolution.

Phénomènes observés après l'extraction du cristallin normal. - Après l'extraction du cristallin normal des yeux, les cristalloïdes se rapprochent ordinairement l'une de l'autre, à l'exception de leurs partics lésées par l'aiguille à cataracte, et s'adossent, pour ainsi dire, sauf les cas oì la substance corlicale cristalliuicnue est restée en place au moment de l'opération. $\Lambda$ près le rélahlissement de la chamhre antérieure, l'augmentation de l'humeur aqueuse et sa pression sur liris qui s'ensuit, ce dernier s'ćloigne de la corncé, prend la forme d'un entonnoir et s'applique contre la capsule cristalliuienne ridée. Au moment de l'incision de la cornéc, de l'évacualion instantinée de la chambre antćrieure el de l'extriction du cristallin, l'iris se trouve gorgé de sang; ct lorsque plus lard la chambre antérieure se rétablit, ce qui a lieu très-vile dans les cas l'opérations réussies, il s'opeère un dégorgement de ce mème iris. Le dégorgement s'accompagnail-il de déchirures de quelques vaisseaux cappillaires de l'iris et de légères cxtravasations? Nous ne salurions l'affirmer. Lorsque l'iris élait enlianmé, mème légèrencut, le produit inflammatoire était surtout manifeste entre le hoid pupillaire de l'iris et la capsulc criślallinienue, correspondait très-sourent au pólc antéricur de l'apparcil cristallinien, c'cst-it-dire, dans ros expériences, il la fossctte annulaire des cristallins régénérés, el était presque loujourssuivi d’un dépôt pig̣nentaire rlel'uvée. On rovail hiculòt après 
apparaitre, soit sur la lossette annulaire, soit entre l'iris et la cristalloïde antérieure, des vaisscanx capillaires et du lissu conjonctif. Malheureusement nous n'avons pu noursuivre et, par conséqueut, nous ne pouvons préciser le moment de la formation des vaisseaux capillaires, ni la fréquuence de l'appirition de ces derniers; mais ce que nous pouvons alfirmer, c'est que cette appa-rition était loin d'être rare.

Dans les expériences à résultat négatif, accompagnées d’inflammation de l'iris, du corps ciliaire, etc., le tissu conjonctif de nourelle formation se développrait non-seulement dans la cavité de la capsule cristallinienne, mais aussi sur sa surface antéricure et concourait à la formalion d'une memhrane épaisse qui a dhérait soit au bord pupillaire de l'iris, soit à la cicatrice cornéenue. Dans les expériences accompagnées également d'inflammation des parties roisines de l'appareil cristallinien, mais à résultat positif, le produit inflammatoire se résorbait et la pupille reprenait sa forme plus ou moins normale; il arrivait cependant assez souvent qu'entre la surface antérieure de la capsule du cristallin régénéré et le bord pupillaire de l'iris et de la cicatrice cornéenne il restait des adlıérences minces, délicates et filiformes.

Les changements qui avaient lien dans la capsule cristallinienne après l'extraction du cristallin, sont uon moins intéressints à signaler que ceux des parlies quil'avoisinent, et que nous venons d'indiquer. Le cristallin, remplissant entièrement la capsule cristallinienne, ne peut en sortir facilement que dans le eas où celle dernière est incisée à sa partic équatoriale sur la distance d'au moins la moitié de sa eirconférence, ou bien lorsque les cristalloijles, antérienre ou postérieure, sont fendues dans différentes directions. Aprés l'ouverture de la capsule cristallinienne, par le procŕdé ordinaire, c'est-il-dire par une incision verticale, trans. versale su rruciale, nous obtenious dans nos expériences des fentre irusuffisautes pour l'extriction du cristallin; aussi celui-ci, perrdant sa sortie lente ou promple, par exemple, lorsque les muscles de l'wil se entrarlaient spasmodiquement ou bien, au momert de la pressinn du gelobe oculaire par la cureltr de Daviel, les agrandiseait, pour ainsi dire, lui-même. Cet agrandissement 
de: léntes de la capsule etait toujours en ritison directe de la vilcsso avec laquelle sortait le cristallin. Doué d'une certaine 'lasticité, le cristallin passail, néanmoins, bien rarement à traver's les feules, relativement petites, de lia capsule cristalliuienne, et encore cela n'avait-jil lieu que lorsque sa sortic était lente. Il est bien entendı que tont ce qui vient d'être dit ne sc rapporte nulIcnent aux cas où le cristallin, pendant sa sortie, laissait dans sa capsale une certaine parlie de sa surface corticalc.

L'agrandisscment forcé des fentes capsulaires, produit par les cristallins ì leur sorlie, avait lieu bicn rarement dans une seule el unique direction. Ainsi, par exemple, la capsule cristallinienne, ouverte au moyen de l'aigutille dans la direction du diamèlre horizontal de l’appareil cristallinien, était trouvée, à l'cxamen du globe oculaire extirpé, ouverte aussi dans la direction du diamètre perpendiculaire du mème appareil (voy. pl. VI, fig. 2). Lorsque, pour la prenière fois, l'agrandissement forcé par le cristallin des fentes capsulaires attira notre attenlion, il nous semblait naturel que cet agrandissement s'étendît non sculement jusqu'à la partie équaloriale de la capsule, mais encore jusqu’à une cerlaine partie de la cristallö̈de postérieure. Cependant, plus tard, nous nous convainquimes que cet agrandissement forcé n'avait licu qu'au détriment de la cristalloïde antérieure et n'aboutissait pas toujour's à la partie équatoriale de cette dernière, par suite peut-ctre de la résistance que lui oppose la zonule de Zinn. N'ayant fait qu'un petit nombre d'observations concernant l'agrandissement des fentcs capsulaires, nous ne pouvons ni ne voulons nous prononcer d'une manière positive sur la forme el l'agrandissement le ces fentes. Nous dirons néanmoins qu'il nous est arrivé d'observer', après l'extraction dilficile du cristallin normal, des fentes à la cristalloüde postérieure el antérieme de la capsule, sans pouroir constater l'existence des fentes équatoriales. Daus ce dernier cas, l'humeur vitrée cédait à la contraction des muscles du glohe oculaire, et ayant devant soi ume ouverture faite ì la capsule cristallinienne, la traversait et apparaissnit soit daus la chambre antérieure vidéc, soit dans la plaie comcenne où parfois clle faisait hernie. Get écoulement de l'humeur vilrće à travers l'onverture capsulaire 


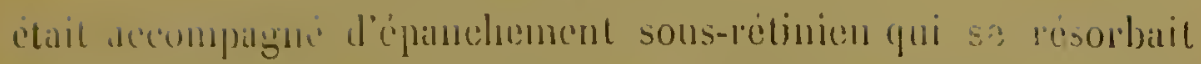

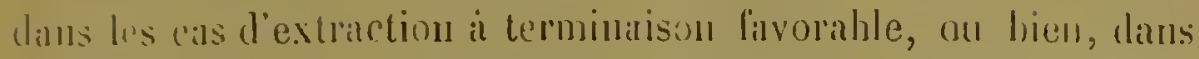
lesopriraticiss il terminaison définorable, produisait le décollement de la retine sur une étendue plus ou moins grande. Ge décollement prenait peu it peu de plus grandes dimensions, comprimait l'humeur vitrie et l'atrophiait; la rétine se ratalinait et prenail, avere l'hyjaloüde, la lorme d'un cordon allant de la papille clu uerf optique ì l'appareil eristallinien, c'est-i-clire à la crisialloüde poslérieure à latguelle êlle ad̉ı́rait.

Pour critcr l'agrandissement forcé des incisions de la cristalloỉle antérieure el rendre ces dernières aussi peu étendues que possible, nous avous tichc, malheureusement trop tard, de leur douner la forme demi-circulaire el corresprondant it l'iucision corméenne. Nous faisions notre incision au niveau du bord pupillaire de l'iris moỹennement dilaté, c'est-à-dire à l'endroil correspondant à l'insertion de la zonule de Zinn à la cristalloïde antérieure de la capsule cristallinienne. Nous devous dire cependant qu'ell procidant ainsi, et malgré tous nos efforts, nous n'ayons pu obtenir que des incisions demi-lunaires. Pour inciser plus commodément la capsule, nous avons cmployé, soil l'aiguille ggcuonillée spécialement confectionnée pur nous par Lücr (roj. pl. I, fiø. 1, b), que nous introduisions dans la chambre antérieure vidéc, parallélement au plan de l'iris; nous relevions ensuite le manche de manière à le rendre perpendiculaire a la capsule, el lui commuliiguions un mouvement de rolation pour faire l'incision demicirculaire, soit l'aiguille le Cheselden, liggèrement recourbée et Irauchauble des deux colís a son hout (rog. pl. I, fig. 1, a). Nons arons trouve ce deruicer iusl rument bicu superrieur il notre aiguille renruillece, dont l'introdıction dans la chambre antérieure n'est paifarile, el don le matement exige non seulcment beauroup i) Jálitule, mais encore une certaine labileté.

Sinns erprirnis fue le cristallin, lans les cas de sorties difficiles,

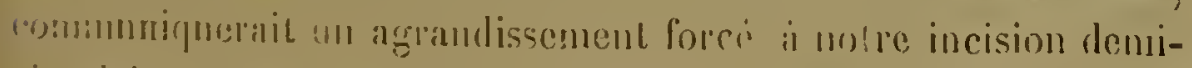

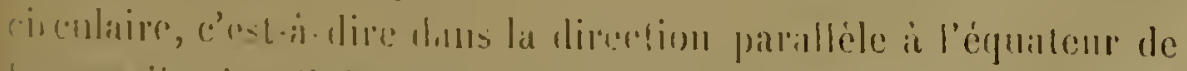

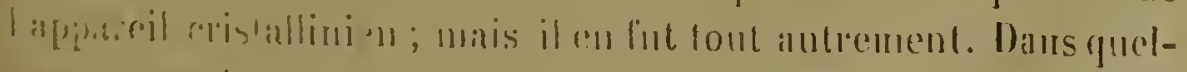

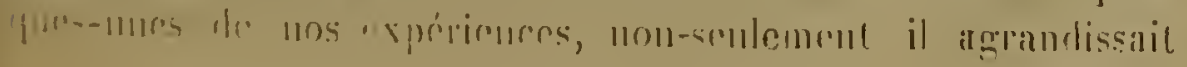


l’incision dans une direction dillérente, mais quelquefois mène dans une direction perpendiculare it la notre. Nous mellons cependant de còté, jusqu’ì de nouvelles expériences, la solution de celte importante question, complant la reprendre plus tard; nous la meltons de côté d'autant plus volontiers, qu'au lieu des incisions demi-circulaires projetées, nous en faisions en réalité de demi-luniaires.

Après l'ouverture de la capsule cristallinienue, par un des pro. cedés mentionnés, les bords de l'cndroit lésé de la cristalloüde : 1. ou bien se recroquevillaient, aprés s'átre éloignes da pôle antéricur, haissant une place vide qu'oceupait la cristallö̈de postéricure, lorsque celle-ci n'élait pas lúsece, ou la membrane de l'humcur vilrie, quand celle dernière réoccupait sal place dans les expériences où son écoulement avail éu lieu. Ces bords recroquevillés, éloignés phus ou moins de l'endroil qui correspond au pôte antérieur do l'appareil cristallinien, restaicnt libres dans les premiers momenls fui suivaient l'opération el permettaient à la nouvelle humeur aqueuse de la chambre anterieure d'agir sur le rontenu de la partie équatoriale intacte de la capsule cristallinieme. Peu de temps cependant aprés l'opération, ees bords adhéraicnt à la cristalloüde postéricure de lia capsule, au moyen d'une substance amorphe ou d'un tissu conjonclif nouvellement formé, el coopéraient par là ì l'occlusion de la partic équatoriale de lia capsule cristallinienne $\mathrm{cl}$ à la formation (dans les cas oú cette deroicre était remplie de substance corticale restéc aprés l'extraction du cristallio, ou d'éléments du cristallin régénéré), de l'anneau cristallinien (Krystallwulst) de Soemmering, de différentes formes el grandeurs, à crnmencer d'un anncan regulier et bien dessiné, el terminant par un bourrelet demi-lmaire occupant une parlie trés-minime de la circonférenee de l'apparcil cris. tallinicu. Nous avons rencontré l'anneau cristallinien régulier bien souvent aprés l'éconlcment de l'huneur vitréc, ct aujourd'hui encore nous doutons que sa formation soil possible sans cet écoulencnt, plus ou moins marqué, à travers la cristalloïde poslérieuro lésće de la capsule eristallinienne; $2^{n}$ ou bien les bords de

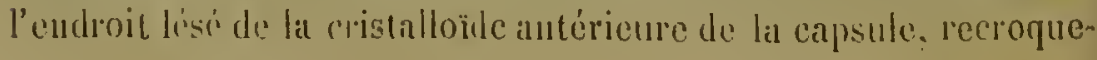




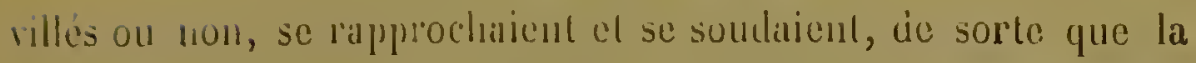
capsule cristallinienne, remplic par le cristallin régénéré, reprenait sa forme primitive el présentait sun ancien aspect. Lat partie de la capsule cristallinienne qui u’élail pas lésée par l'aiguille, c'est-i-dire surtout sa partic équatoriaie, contenail dans la suite de l'opération : $a$. ou de l'épithélium de la capsule cristallinienne et des tubes cristalliniens, en $110 \mathrm{mb}$ re plus ou moins grand selon qu'était plus ou moins difficile la sortie du cristallin; $b$. ou un liquide amorphe el hyaliu, de l'épithélium de la capsule et des tubes ot fibres eristalliniens ayant subi, dans les cas où ils étaient en petite quantite, la transformation granulcuse (voy. pl. III, fig. 2 et 3) ; ou enfur $c$. un liquide amorphe, des globuies de sang el des parties de l'humeur vitríe dans les cas d'écoulentent de celle dernicire.

Nous ne saurions allribucr la transformation granuleuse des tubes et fibres cristalliniens uniquement à l'influence de l'humeur aqueuse, parce que, dans une de nos cxpériences, nous trouvâmes, chez un vieux chien il est vrai, 144 jours aprés l'opération, des fibres cristalliniennes en transformation granuleuse (voy. pl. III, fig. 3,e).Quant à ce qui concerne l'épithélium de la capsule, ce dernier restait intact et adhérent à la surface de cetle dernière, dans les cas ou l'extraction du cristallin s'opérait lentement el sans difficulté ; mais dans les cas où la sortie du cristallin se faisait rapidement, l'épithélium était arraché par endroits de la surface intérieure de la capsule cristallinienne, et entrainé au dehors avec le cristallin sur lequel on pouvait l'apercevoir à l'œil nu, en forme de petites taches demi-Iransparentes.

C'e que l'on doit cntendre par cristallin régénéré. - Dans l'intérieur de la partie équatoriale de la capsule cristallinienne, les cellules formatrices de Becker donmaient naissance aux tubes cristalliniens par leur alignement en séries linéaires (voy. pl. V, fig. $2, b)$, exactement comne dans les yeux des embryons ou de trés-jeunes ánimaux (1). A côté de la déconverte, faite par

1) Voyez llislnire du développement de l'ail humain, par F. A. d'Ammon (dnnales d'oculistirue. 1860 ). 
Meyer (1), de la zour nucléaire (Kernzone, voy. 11 l. l, lig. 5, b), celle de F. J. Becker, prof'ssener ì Holsinglors, des cellules formatrices (Bildungszellen), de lit partie infartorialte de la capsule cristallinienue, oecupera une place importante lans l'histoire de la strueture de l'appareil eristallinien (2). Tout en passant sous silenee, comme n'entrant pas dans le plan de ce travait, la question de la formation des luhes cristallimins el lesopinions, à eet égard, de divers sivants entre autris dir Balontilne (3), do Zeriow (4) et d'Lwanow (5), nous devons dire que uous parlageans l'opinion de Beckrr, d'ar̃rès laquelle les cellules formatrices de cet auteur donnent uaissance aux (ubes cristalliniens. Dans nos expériences nous avons vu, maintes fois, lcur alignement par séries linéaires. Ians les cas oú, aprés l'opération, il resail dans la eapsule cristallinienne une certaine quantilc de substance corticale, eet alignement avait igalement lieu, ot les cellules formatrices étaient en nombre plus oumoins grand selon yue ia yuantilé des vaisseaux eapillaires, de nouvelle lormation, disseminés sur la surface antérieure de la cristalloïde antérieure, était plus ou moins forte.

Nous arons dit qu'entre l'iris el la capsule cristallinieme il se formait du tissu conjontetil et les vaisseaux capillares disséminés sur la surface antérieure de celle deruière (jl. V, fig. $2, d)$. L'apparilion de ces vaisseaux capillaries ce l'augmentation de la vilalité du corps ciiaire el te l'iris nous expliquent pourquoi l'aetion des eellules

(1) *II. Mever, Beit?ag zu der Strciffrage ueber die Enlwiclehung der Linsenfasern (Arch. F. Analomie und Physiologie, 1851).

(2) F. J. von Becker, Untersuchungen ueber den Bau der Linse bei dem .Jenschen n. den Wirbellhieron (Archiv f. Ophlhalmologie v. Arll, Donders und v. Graefe. Bd. IX, liten Abth. 1863).

(3) * Bubouchin, Beilrilge zur Enlwicliclungsgeschiche des Auges (Wiirzb. Nalurwissensch. Zeilsehr. 1864).

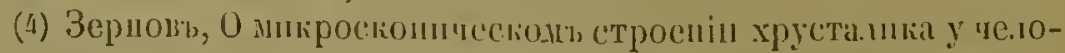

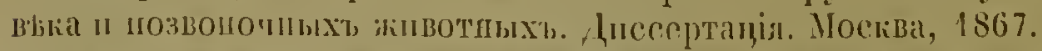

Zum miliroscopischen Bau der Linse beim Menschen u. bei den Wirbelhieren, $v$. Zernoff in Hoskau. (In Archiv f. Ophthalmologic v. As:ll, Donders u. v. Gracfe. 1867, T. Xill, Abth. II, p. 521-546.)

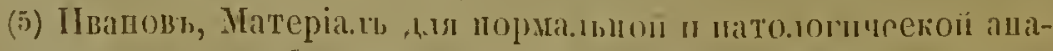

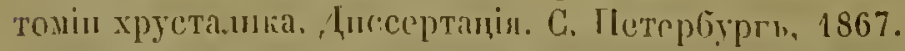

Iranow, Malériaus pour servir a l'analomic normale ol pathologique du cris. tallin. Thèse inaugurale. Suint-l'élershourg, 1867. 
Cormatrices devient plus grande el pourquoi la régénération du cristallin a lieu relativement assez vite. Comme preuve que la régénération du cristallin est en raison directe de l'augmentation de la vilalité des organes environnants, nous citerons ce fait que, dans certains cas de nos expériences où la réaction de ces organes était nulle et la terminaison de l'opération très-favorable, la régénération du cristallin n'avait néanmoins pas lieu.

Nous avons déja dit plus haut que dans le cas où, aprés l'extraction du cristallin normal de sa capsule, il en restait dans celte dernière quelques partics, celles-ci subissaient la transformation granuleuse; mais là où le nombre des tubes cristalliniens, restés dans la capsule, était en quantité tont soit peu notable, ils continuaient d'exister sabs subir cette transformation et faisaient mème partie du cristallin régénèé, lorsque cette régénération arait licu. Dans d'autres cas moins favorables, ces mêmes tubes devenaient plus larges, c'est-à-dire s'hypertrophiaient.

Circonstances défavorables à la régénération du cristallin. Aprés ayoir décrit, dans des termes généraux, l'évolution du cristallin régénéré, nous devons mentionner, en passant du moins, les circonstances défavorables au milieu desquelles se trouvait, dans nos expériences, la capsule cristallinienne aprés l'extraction des cristallins. Si mème nous mettons de côté les lésions étendues de la capsule cristallinienne, les phénomènes inflanmatoires des organes qui l'environnent et l'épanchement qui s'ensuit, écrasant lour aiısi dire sa vilalité, il suffit de mentionner : l'adhérence qui si solvent a lieu entre les bords de l'endroit lésc de la cristalloïde antérieure avec la cristallö̈de postérjeure, la pression que suljit, après l'opération de l'extraction, la cajsule de la part des liumeurs vitrées et aqueuses, et cufin l'absence de l'augmentation de l'activité de l'iris et du corps vitré aprés un certain laps de tempes écoulé depuis l'opération, activité si nécessaire pour accélérer lá formalion des éléments du cristallin, pour comprendre les difficultés que doit surmonter l'appareil cristallinien, pour arriver à un cristallin regenere; et cependant la loree régénératrice de la cápsule cristallinienne est si grande que malgré toutes res entraves clle régénére le cristallin, et jusqu'it tel point, que

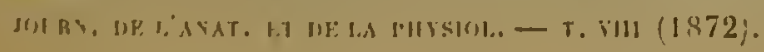


s'il n'atteignit jamais dans nos exproriences le volume du cristallin normal, il acquit dans l'une d'elles la moitic de son volume, et dans une autre il la dépassa. On comprend que dans ees cas les amimax pouvaicnt reconquérir plus ou moins la vue normale, et, on elfel, ils la reeonquéraient.

L'examen microscopique de la partic de l'apparcil cristallinion, restéc dans l'oxil après l'extraction du eristallin normal, démontra:

$1^{\circ}$ Dans les eas oú la régénération du cristallin arait lieu, et surtout dans ecux oì l'animal était sacrifié plus ou moins longtemps après l'opération, non-seulement l'existenee des mèmes éléments anatomiques que dans l'apparcil eristallinien normal, mais encore une disposition analogue à celle des éléments eristalliniens normaux; ạnsi nous avons eonstaté dans ees eas: l'épithélium, les eellules de Robin (pl. III, fig. 3, c), la zone nuelćaire de IIeyer (pl. I, fig. $5, b)$; les tubes et fibres eristalliniens avee les espaees interfibrillaires (pl. IV, fig. $3, c, e^{\prime}$ ); cependant, dans la plupart des cas, ces derniers éléments étaient plus g̨rands qu'à l'état normal.

2. Dans les eas où la régénération du eristallin avail lieu, mais où les animaux étoient sacrifiés bientôt après l'opération : de l'épithélium de la eapsule eristallinienne ì l'état normal (pl. III, fiğ. 3,6 ) des eellules de Rohin (pl. III, fig. 3, c, et fig. 4, c), de tubes cristalliniens restés, après l'extraction du cristallin, normaux ou modifiés et ayant la forme de massue eourle ou de matras (l. I, fig. 2, e, et pl. II, fig. 1, b), de globules de Morgagni (pl. VI, fig. "3, g), enfin de cellules formatrices éparses dans un liquide hyalin ou agglomérées et alignées par sérics linéaires ( $\mu$ l. III, fig. 3, d, fig. il, $f, d$, et pl. V, fig. $2, b$ ); ees dernières s'observaient quelquefois dans les eas où la eapsule cristallinienne ne contenait ni tubes, ni fibres cristalliniens.

$3^{\circ}$ Dans les cas où la régénćration du cristallin u'avait pas cu lieu : du lissu conjonctif de nourclle formation (p!. 1, fig. 2, c, et pl. III, lig. 1, a), de vaisscaux capillaires, de gramulations, de grains et de cellules pigmentaires (pl. V, fig. 2, a), de grobules de sang et de pus, de eristanx d'hématuïline, de cellules du corps 
vitri rappelaut les globules de pus (pl. VI, fig. 2, b), de l'épithélium de la capsule, de cellules formatrices ordinairement éparses, et de cellules fusilormes (pl. II, fig. $1, f, f)$; dans quelques cas les ccllules formatrices étaient alignécs par séries linéaires et supplantaient, pour ainsi dire, les éléments étrangers à la cipsule, en les atrophiant, graice à la force d'organisation dont ulles sont louces.

Après aroir fait ressortir ces trois formes dislinctes de structure des apparcils cristalliniens, avec cristallins r'égénérés ou nun, nous derons dire que leur structure nicroscopique ne présculait pas tonjours des differences anssi tranchées, et qu'il arrivait bien souvent que les ćléments sus-mentionnćs, tous ou en partic, existaient en mème temps dans la capsule cristallinienue.

\section{III}

\section{EXPÉRIENCES DE L'AUT'EELI ET CONCLUSIONS。}

\section{Expériences.}

Lapins. - Des 19 expériences failes sur des lapins, '7 (1, 5, $10,11,14,15$ et 1S) eurent un résuliat posilif, c'est-i-dire présentérent des cristallins plus ou moins régénérés; et $12(2,3,4$, $6,7,8,9,12,13,16,17$ ct 19) eurent un résultat négatif.

1. Jans la $1^{\text {re }}$ expérience, à résultat posilif, à un lapin de 3 mois, il fut fait la liératotomie supéricurc sur l'oxil gauche, 'fui fut suivie de la procidence de l'iris. L'examen de l'œil, fail 451 jours (du 23 juin 1862 au 16 scptembre 1863 ) après l'opératinri, y déniontra l'existence d'un cristallin régénéré, transparent, aplati, et de la grandeur du tiers du cristallin normal; sa capsule contenait des tubes el des fibres cristalliniens; la démonstratirn de cet wil fut faite à la Socićté des médecins de Kiew, dans la scarce du 21 creptembre 1863.

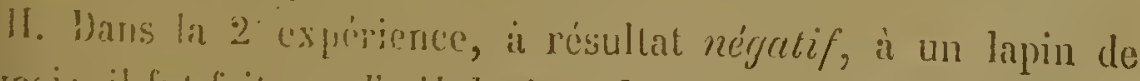
I mois, il fut fitit sur l'eril droil la liératolomic supéricure, que suivit la procidenee et l'etranglement le l'iris dans la plaie; l'examen de l'rsil, fail 395\% jours (du 2/ juin 1862 alu 23 juillet 2863 ; 
iprès l'operation, n'y montra pas la présence du eristallin régénéré.

III. Dans la $3^{*}$ expérience, à résultal négatif, à un lapin de 4 mois, il ful lait, sur l'ail gauche, la kiératotomie supérieure que suivit la procidence de l'iris ; l'examen de l'œil, lail 419 jours (du 24 juin 1862 au 16 août 1863) après l'opération, y démontra la présence d'une simple membrane, à la place du cristallin l'égénéré.

IV. Dans la $4^{\mathrm{e}}$ expérience, à résultat négatif, à un lapin de 3 mois, il fut fait sur l'œil droit, la liératotomie supérieure que suivit la proeidence de l'iris l)lessé; l'examen de l'oil fail 391 jours (du 24 juin 1862 au 19 juillel 1863) apris l'opération, démontra l'atrophie du globe oculaire.

V. Dans la $5^{\circ}$ expérience, à résultat positif, à un lapin de 3 mois il ful fail, sur l'œil ganche la lératotomic supérienre; l'examen de l'œil, fait 427 jours (du 26 juin 1862 au 26 août 1863) après l'opération, y démontra la présence d'un eristallin régénéré, ayant la forme d'un annean épais, large et transparent, avee une petite fosselle annulaire transparente; l'anneau cristallinien coiltenail des tubes el des fibres eristalliniens.

VI. Dans la $6^{\circ}$ expérienee, à résultat négatif, à un lapin de 5 mois, il fut fail sur l'œil gauche la kératotomie supérienre, qui fut suivie de l'expulsion spontanée du eristallin, par suite de la contraction spasmodique des muscles de l'œil; l'examen de ce dernier fait 424 jours (du 6 juillet 1862 an 3 septembre 1863) après l'opération, y démontra l'absence d'un cristallin régénéré.

VII. Dans la $7^{\mathrm{c}}$ expérience, à résultat négatif, à un lapin de à mois, il fut fail sur l'œil droit la kératotomic supérienve ; l'iris fut blessé, et il y eut éeoulement d'une eertaine quantité de l'humeur' vitrée; l'examen de l'œil, fail 435 jours (du 6 juillel 1862 au 14 septembre 1863) aprés l'opération, y démontra à la place de l'appareil cristallinien la presence d'une membrane dure el épaisse.

VIII, Dans la $8^{0}$ exporience, à risullat nérgatif; à un lapin de 5 mois, il fut fail sne l' ail yauche lis liératotomie supérieure, il y eu! blessure et procidence de l'iris; l'examen de l'ceil fail 
429 jours (clu 13 juillet 1862 an it septembre 1863 ) après l'opération, en clémontra l’atrophie.

IX. Dans la $9^{\mathbf{c}}$ expérience, à résultat négatif, à un lapin de ó mois, il fut fait sur l'ail gauche la liératolomie supérieure, qui fut suivie de la procidence de l'iris et de l'écoulement d'une certaine quantité de l'humeur vitrée; l'examen de l'œil fait 426 jours (dı 16 juillet $1 \$ 62$ au 14 septembre 1863) après l'opération, y démontra la présence, à la piace de l'appareil cristallivien, d'une membrane épaisse et opaque.

I. Dans la $10^{\mathrm{e}}$ expérience, ì résultat positif, à an lapin de 3 mois, il fut fait sur l'wil gauche la kératotomie supérieure, qui' fut suivie de la procidence peu marquée de l'iris ; l'examen de lœeil, fait 402 jours (du 24 juillet 1862 au 29 août 1863) après l'opération, y démontra la présence d'un cristallin régénéré, dont la dimension égalait la moitié de celle du cristallin extrait; il avait la forme arrondie et élait un peu aplati l'avant en arrière; il élait transparent, el, plongé dans l'alcool, il devint opaque; il était constilué par des tubes et fibres cristalliniens. Ce cristallin régénéré fut présenté à la Société des médecins de Kiew, dans la séance du 21 septembre 1863 ..

XI. Dans la $11^{\mathrm{e}}$ expérience, à résullat positif, à un lapin de 4 mois, il fut fait sur l'ceil gauche la kératotomie supérieure; l'examen de l'ail, fait 414 jours (du 28 juillet 1862 au 14 septembre 1863) après l'opération, y démontra dans la partie inférieure de la capsule cristallinienne, un bourrelet demi-lunạire; à la partie supérieure de la capsule, les cristalloides étaient adhérentes l'une a l'autre.

XII. Jans la $12^{\mathrm{c}}$ expérience à résultat négatif, à un lapin de I mois, il fut fait sur l'ceil droit la kératotomie supérieure; l'examen de cet ceil, fait 413 jours (du 29 juillet 1862 au 14 septembre 1863 ) apres l'opération, en démontra l'atrophie.

XIII. Jlans la $13^{\mathrm{e}}$ expérience, it résultal négatif, à un lapin de 5 mois, il fut fait sur l'ceil droit la kératotomie supérieure; l'examen de cet rsil, fait 1.47 jours (du 9 novembre 1865 au \ avril 1866) aprés lopération, y démontra la présence de la capsule cristallinienne, dont quelques parties senlement n'étuient 
pas transparentes, et dont les cristallö̈des étaient rapprochées l'une de l'antre; l'anneau était faiblement accentué, sa losselte élait irrézulièrement ovale, transparente et reeonverte de pigment dans un endroit ; l'annean contenait du tissu conjonetif entièrement formé (prés de la f'osselte), de l'épithélium de ła capsule, des cellules formatrices éparses el des tubcs cristalliniens plus gros qu'il l'état normal et en certains endroits ayant la forme de massue (voyez pl. I, lig. 2).

XIV. Dans la $14^{\circ}$ expérience à résultat positif, à un lapin de 5 mois, il fut fint sur l'ccil droit la lieratolomie supérieure, qui fut suivie de la proeidence de l'iris hlessé; l'examen de eet oil, fait 73 jours (du 19 mai au 30 juillet 1S66) après l'opération, y démontl'a l'existence d'un cristallin rćgénéré, de la grandenr d'un tier's du cristallin normal. Il élait transparent, mamelonné el aplati d'avant en arrière, surtout dans la direction du diamètre horizontal; les parties supérieure el inférieure de la capsule eristallinienne, l’inférieure surtout, étaient asscz épaisses; la capsule contenait: de l'épithćlium, des tubes cristalliniens, un peu plus gros qu'à. l'état normal, mais disposés d'une manière régulière, et des fibres cristallinicrmes (voy. pl. I, hig. 5).

XV. Dans la $15^{\circ}$ expérience à r'ésultat.positif, il in lapin de $S$ mois, il fut fuit sur l' xail guuche lá liérutotomie supérieure; l’iris fut légèement blessé; l'examen de ect œil, fait 11 jours (du 17 au 27 septembre 1866) après l'opération, y démontra la présence d'une capsule cristallinienne transparente; son eontenu élait gélaliniforme, et ses erislalloüdes, excepté la partic de la crislalloïde antérieure lésée par l'aiguille, étaient assez rapproelrées l’une de l'autre; la capsule eontenait : de l'épithélium, des cellules formatrices avec des noyaux disposés en séries réğulières, des tubes cristalliniens normaux et modifićs arec des bouts pyriformes, des granulations et du piginent.

XVI. Dans la $16^{\circ}$ expérience a résultat négatif, à un lapin de 8 nois, il fut liail sur l'ceil droit la liéralotomic supéricure; l'iris fut blessé el le cristallin expulsé : pontanément par suite de lu eontraction spasmodigue des muscles de l'œil. It y eut su mene temps reoulement d'une partie de l'lument vitrée; l'examen de 
l'ail, finit 29 jours (du 17 septembre an 15 octobre 1866) après l'operértion, y démontra l'athérence des eristalloüdes, au moyen d'un tissu conjonclif de nouvelle formation; ; ce tissu ne contenait pas de tuhes cristalliniens, mais dans la partie équatoriale de la capsule on trouvait par endroits des cellnles lormatrices.

IrII. Dans la $17^{\circ}$ expérience à résultat négatif, à un lapin de 9) mois, il fut frit sur l'cil gauche la hératotomie supérieure; l'examen de cet oil, fait 5 jours (du 11 au 15 octobre 1866) aprés l'opération, démontra dans la capsule cristallinienne du pus et les cellules formatrices, nageant dans le liquide purulent. L'épithélium de la capsule n'était conservé que par endroils, mais les Lubes et fibres cristalliniens avaient disparu.

XVIII. Dills la $18^{\circ}$ expérienec à résultat positif, à un lapin de 4 mois, il fut fait sur l'œil droit la liératoiomie supérieure; l'iris fut blessé et le cristallin extrait avec diffieulté ; l'examen de l'œil fait 46 jours (du 9 janvier au 23 février 1867) a près l'opération, y démontra l'existence d'un cristallin régénéré, formé par deux bourrelets, dont l'inférieur était plus grand et le supéricur plus petit; ils élaient séparés par une fosselte annulaire, transparente cl ovale dans la direction du diamètre horizontal de l'appareil cristallinien; le bourrelet supéricur adhérait par son milieu à l'iris; l'appareil cristallinien contenait des tubes et fibres cristalliniens, les uns nornaux, les autres fortement grossis, de l'épithélium et des glolules de Horgagni (royez pl. III, fig. 1).

XIX. Dins la 19'expérience ì résultat négatif, à un lapin de 6 is 8 moir, il l'ut lait sur l'ceil gauche la kératotomie inférienre, qui fut suivic d'un écoulement de l'lumeur vitrée, dù ì la contraction spasmodique des muscles oculaires. L'examen de l'œeil lanit 40 jours (du 9 junvier au 23 février 1567) après l’opération, en dénontra l'atroplic.

Chiens. - Jes 17 expuériences fuites sur des eliens, 5 (22, $26,27,29$ et 33$)$ curent un risullat posilif et $122(20,21,23$, $24,25,28,30,81,32,34,35$ ct 36 ) eurent un "cstetct nigatip.

Xi. Dans la 20 exprerience it résultat mérgaly, i lin chien de 2 an-, il fut lait sur l'wil gauche la licralotomic stpericure, yu fut

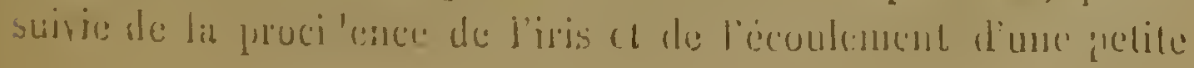


quantité d'humeur vitrée; l'examen de l'cil, fait 42 jours (du 22 juillet au ${ }^{10 r}$ septembrc 1866) après l'opération, y démontra la préscnce d'un bourrelet mince, transparcnt, irrégulier el bosselé au voisinage de la fosselte cristallinienne; cette dernière était recouverte par une sulsstance amorplie, granuleuse et pigmentće, du tissu conjonctif el du pigment; la partie postérieure de la fossette cristallinienne et de l'anneau cristallinien avoisinant adlié.. rait à la rétinc, transformée en un cordon se dirigeant de la papille du nerf optique vers eux; l'anneau contenait en pctitc quantité des tubcs cristallinicns, de l'épithélium, des cellules folmatrices, et en grande quantité des globules de Morgagni.

XXI. Dans la $21^{\circ}$ expérience à résultat négatif, à un chien de 2 ans, il fut fait sur l'œil droit la kératotomie supérieure avcc iridectomie, que suivit la sortie spontanée du cristallin et l'écoulement d'une partie de l'humeur vitrée ; l'examen fait 42 jours (du 22 juillet au $1^{\text {er }}$ septembre 1866) après l'opération, y démontra l'adhésion des cristalloïles au moyen du tissu eonjonctif, au milieu duquel on reneontrait des cellules formatrices. La capsule cristallinienne adhérait fortement par sa face antérieure à une membrane produite par l'inflammation de l'iris.

XXII. Dans la $22^{\circ}$ expérience à résultat positif, au même chien (de 2 ans), de l'expérience précédentc, il fut fait sur l'œil gauche la kératotomie supérieure, qui fut suivie de la procidence de l'iris et de l'écoulement d'une partie dc l'humeur vitrée; l'examcn de cet ceil, fail 42 jours (du 22 juillet au $1^{\text {er }}$ septembre 1866) après l'opération, y démontra la présence d'un eristallin régénéré ayant la forme d'un fer à chcval ct dont le bourrelet aplati avait 2 millimètres ct demi d'épaisseur; la fossette annulaire était ovale, dans la direction du diamètre perpendiculaire de l'appareil cristallinien, transparcute et adhérente à l'iris, à l'endroit où le bourrclet ćtail interrompu. Au côtć opposé à celui de l'adhćrence à l'iris, les rebords de la cristalloïle antérieurc produits par les lésions qu'avait fiites l'aiguille ì cataracte, formaient deux fissures; le bourrelet cristallinien contenait des lubes et fibres cristalliniens, de l'ćpithélium polyédrique et eylindrique, des cellules de Rolin et des cellules formatrices (voyez pl. II, lig. 2). 
¿.111. Dans la -23e expérience à résultat négatif, à un chicn d'environ 3 ans, il fut liat sur l'ail yauche la kératotomie supérieure; l'cxamen fait 51 jours (du 22 juillet au 10 septembre 1866 ) après l'općration, y démontra la préscnce d'un hourrelet peu accentué, surtout au cóté externe où il adlı́rait à l'iris et à la cicatrice de la cornée; le bourrelet contenait : de l'épithélium normal, des ccllules de Robin. des cellules formatrices, du tissu conjonctif de nouvelle formation, des cellules fusiformes et des tubes cristalliniens ayant la forme ampulaire (voy. pl. III, fig. 1).

XXIV. Dans la $24^{\mathrm{e}}$ expérience à résultat négatif, ì un chien de 3 ans, il fut fait sur l'cil gauche la lératotomie supérieure; l'examen de cet ceil fait 4 jours (du 23 au 26 juillet 1866) après l'opération, y démontra l'adlhérence intirne des cristalloïdes; la cristalloïde antérieure était rccroquevillée à l'endroit de sa lésion et opaque, par suite d'une membrane occupant sa surface antérieure; la cavité de la capsule crislallinienne contenait : une substance granuleuse amorphe, de l'épithélium capsulaire, des cellules formatrices éparses, des tubes cristalliniens grossis, recourbés, a bout pyriforme et ayant subi une transformation granuleuse, et des globules de pus (voyez pl. III, fig. 2).

XXV. Dans la $25^{\text {e }}$ expćrience à résultat négatif, à un chien de 2 ans, il fut fait sur l'œil droil la kératolomie supéricure; l'extraction du cristallin fut faite avec difficulté; l'examen de l'œil fait 48 jours (du 3 août au 19 septembre 1866) après l'općration, démontra que les cristalloïdes transparentes adhéraient l'une à l'autrc, et que l'anneau cristallinien, faiblement accentué, était interrompu dans plusieurs endroits et présentait des stries allant dc la fossctte, irréguliẻrement ovale et pigmentée à sa partie suprírieure, a la périphériè de l'appareil; la cavilé de l'anneau eonlenait de l'épitlélium normal, des ccllules de Rohin, une substance granuleuse, des cellulcs formatrices éparses ct des globules de Morgagni.

XIVI. I)ans la $26^{\mathrm{e}}$ expérience à résultat positif, à un chicn de 6 rnois, il fut fait sur l'ceil gauche la kératotomie supérieure; l'iris fut blessé ct le cristallin extrait avec difficulté; l'examen de cet (sil, fait 16 jours (du 13 an 28 août 1866) après l'opération, y dé- 
montra la présence d’un anneau cristallinicn faiblement accentué et d'une fossette cristallinienne ovale; la cavité de l'anncau contenait : de l'épillı́lium, des tubes el des filsres cristalliniens, des cellules formatrices i noyaux alignés par séries linéaires et éparses el des globules de Morgagni en grand nombre.

XXVII. Nans la $27^{\circ}$ expérience ì résultat positif, à un chien de 2 i 3 ans, il ful fait sur l'ouil gauehe la kératotomie supérieure avec iridectomic; l'examen de cet oeil, fait 29 jour's (lu 13 août au 10 septembre 1S66) après l'opération, y démontra l'existence d'un cristallin régénéré, transparen!, ayant un vingtième du poids du cristallin normal et constitué par de l'épithélium, des tubes et fihres cristalliniens et des ccllules formatrices.

XXVIII. Dans la $2 S^{\circ}$ expérience à résultal négatif, à un chien de 8 à 10 ans, il fut fait sur l'xil gauehe la kératotomie externe avec irideclomic; l'examen de cet $\propto$ il, fait 144 jours (lu $1^{\text {er }}$ septembre 1566 au 22 janvier 1867) aprés l'opération, démontra que les bords de la lésion par l'aiguille de la cristalloüde antérieure étaient adhérents à la cristallö̈de postérieure au moyen d'une substance amorphe; la fosselte cristallinienne élait irrégulièrement triangulaire, transparentc el forméc uniquement par la cristalloïde postérieure de la capsule cristallinienne; l'arneau cristallinien, de 1 1/a millinètre d'épaisseur, contenait : de l'épilhélium polyédrique el des cellules formatrices aggglomérécs dans certains endroils et éparses en l'autres, des tubes cristalliniens ayant subi la transformation granuleuse el des cellules de Robin; la démonstration de cclle préparation a cté faite à l'Académie de médecine de Paris, dans la séunce du 29 janvier 1867 (voyez pl. Ill, fig. 3).

XXIX. Dans la $29^{\mathrm{e}}$ expérience à résultal positif, à un chien de 2 ans, il fut lait sur l'éil guuche la liéralotomic supéricure avec iridectomie; l'examen de cel reil, fail 9 jours (du 11 au 19 septembre 1860̈) après l'opération, ý démontra l’adhérence de l'endroit lésé de la cristalloüde autcricure ì la partie supérieure de l’iris el a la cicatrice cornécnne ; les bords de la lérion de la cristalloïde antericure étaient recroquevilles el adherents atux confins de la

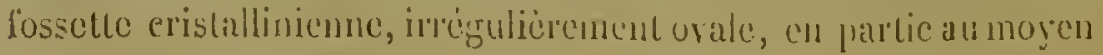


d'une substance amorplıe, en partie all moyen du tissu conjonclif de nouvelle formation; à leur voisinare, mais lans l'intérieur de l'anneau, on voyait des tubes cristalliniens ayant subi la transformation granulcuse; la surface antérieure de la cristalloüde postérieure de l'anncau cristallinien était reconverte, dans la région équatoriale de la capsule, de cellules ovales de Robin, dont partaicnt des tubes cristalliniens modiliés dans la partie équatoriale de l'anneau; à côté de l'épithélium, se trouvaient des cellules formatrices, alignćes en ccrtains endroits, el éparses en d'autres (royez pl. III, fig. 4).

XXX. Dans la $30^{\circ}$ expérience à r’ésultat négatif, à un chien de 2 à 3 ans, il fut fait sur l'ail droit la kéralotomie inférieure; l'iris fut blessé ; l'examen de l'cil, fait 126 jours (du 19 septembre 1866 au 22 janvicr 1567) après l'opération, y démontra la présence de la capsule cristallinienne transparente, divisée du liaut en lias en deux parties inégales, dont l'une, l'externe, était plus grande, et l'autre, l'interne, élait plus petite. Entre ces deux parties apparaissait l'humeur vitrée transparente; la cavité des deux parties, surtout celle de la partie externe, contenait des fibres cristalliniennes normales, minces el aggloniérées dans certains endroits, de l'épithelium, une substance granuleuse et des cristaux d'hémalö̈dine (voycz pl. IV, fig. 1).

XXXI. Dans la $31^{\mathrm{C}}$ expérience à résultat négatif, à un chien de 2 ans, il fut fait sur l'œil ganche la liératotomie supérieure; l'cxamen de l'œil fait 33 jours (du 19 septembre au 21 octob) 1866) après l'opération, y démontra l'adlićrence des cristalloüles de la capsule cristallinicnne, ct celle de celtc derniére avec le bord papillaire de l'iris au moyen du tissu conjonclil de nouvelle formation; entre les deux cristallö̈des, on distinguait par endroits des cellules formatrices, épilhéliales el fusiformes.

XXXII. Wans la 320 expérience ì résultat négulif, à un chien d'un an, il fut fait sur l'ceil ganche la liératotomiesupériezre; ì la suite de l'insulfisance de la plaie cornéenne, le cristallin fut extrait far morceaux el il y eut éconlenent de l'humcur vitréc; l'exanen de l'rejil, fait 20 jours (du z 1 septembre an 10 octobre 1860) apres

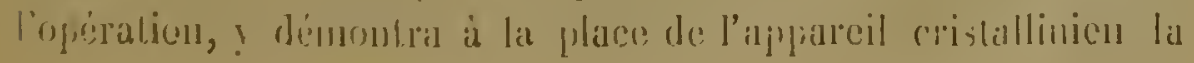


présence d'une membrane opaque, épaisse el constituée par d tissu conjonctil au sein duquel il était difficile de distinguer les cristalloüdes.

XXXIII. Dans la $33^{\mathrm{e}}$ expérience à résullat positif, à un chien de 4 ans, il fut fail sur l'ocil droil la liératotomie supérieure; l'extraction du cristalliu fut faite arec difficulté; l'examen de l'œil, fait 8 jours (du 16 au 23 novembre 1866) après l'opération, y démontra que l'incision de la cristalloüde antérieure était T-forme; la cavité de la capsule cristalliuienne, dans sa partie équatoriale, contenait des cellules formatrices rangées parallèlement à son équateur et d'autres alignées dans la direction de cet équateur au çentre de la capsule par sérics linéaires; près des bords lésés de la capsule, on trouvait une substance gélatiniforme amorphe avec quelques cellules formatrices, des granules de pigment et des cellules granuleuses du corps vilié (royez pl. IV, fig. 2).

XXXIV. Dans la $34^{\circ}$ cxpérience à résultat négatif, à un chien de 3 ans, il fut fait sur l'ceil droit la liératotomie supérieure; l'cxamen de cet oil, fail 8 jours (du 16 au 23 novembrc 1866) après l'opération, y démuntra l'adhésion l'une à l'autre des cristalloïdes, au moyen d'un tissu conjonctif nouvellement organisé et formant, sur la.surface antérieure de la eapsule, et entre les bords pupillaires de l'iris, une membrane opaque; entre les cristalloïdes et à la parlie équatoriale de la capsule, on trouvait par endroits des cellules formatrices el des tubes cristalliniens ayant subi la trans. formation granuleuse:

XXXV. Dans la $35^{\circ}$ expérienec à résultat négatif, à un ehien de 3 ans, il fut fait.sur l'œil gauche la kératotomie externe; l'examen de cet oil, fait 177 jours (du 3 janvicl au 28 juin 1867) après l'opération, en démontra l'atroplice.

XXXVI. Dansla $36^{\circ}$ expérience à résultat négatif, à un chien de 4 ans, il ful fait sur l'œil droit la liératotomie supérieure; l'examen de cet oil; fait 24 heures (du 19) au 20 février 1567) après l'opération, démontıa que les eristailoödes adlıéraient l'une à l'autre, et que la capsule contenait de l'épithélium, des tubes cristalliniens normaux el modiliés, et à bouts pyriformes, des grlobules de Morgagni, par endroits aggloméré, par d'autres épar's, et des globules de sang. 
bíliers et brebis. - Des 1 expériences faitcs sur 2 béliers et 2 brebis, 2 (37 et 40) curcnt nur résullat positif, ct 2 (38 cl 39) un résullut négatif.

XXXVII. Dans la $37^{\circ}$ cxpérience ì résultat positif, à un bélier de Is mois, il fut fait sur'l'ail droit la kératotomie externe, par suite le l'insuffisance de la section cornécune; le cristallin fut extrait arec une grande difficulté ; l'cxamen de cet œil, fait 37 jours (du z mai au 12 juin 1S66) aprés l'opération, y démontra la présence d'un cristallin régénéré de la grandeur de deux tier's du cristallin normal (rapport 31 : 45) ; lc nouveau cristallin élait transparcnt, arrondi à sa partie équatoriale et aplati dans la direction du diamètre antéro-postéricur; la disposition de scs couches était concentrique comme dans lc cristallin normal ; il contenait des tubes et des fibres cristalliniens et des espaccs intcrfibrillaires formés post mortem, comme à l'état normal, mais un pcu plus grands, des cellules épithélialcs, cı des cellules de Rohin. Cette préparation a été démontrée à l'Académie de rnćdecine de Paris, dans la séance du 29 janvier 1867 , et à la Société des médecins russcs, de Saint-Pétersbourg, à la séance du $\mathbf{I}^{\text {er }}$ avril $186 S$ (voyez pl. IV, fig. 3, A ct B). .

XXXVIII. Dans la $3 S^{e}$ expérience i résultat négatif, à une brebis de 2 à 3 ans, il fut fail sur l'oeil gauche la liératotomie externe; l'incision cornéenne fut agrandie au moyen de petits ciseaux; l'examen de cet oil, fait 261 jours (du 7 mai 1866 au 22 janvier 1867) après l'opéralion, en démontra l'atrophie.

XXXIX. Dans la $39^{\circ}$ cxpéricnce à résultal négatif, à unc brebis de 18 mois, il fut fait sur l'oeil gauche la liératolomie supérieure; l'iris ful blessé, el par suite de l'insuffisance de l'incision cornéenne, le crisłallin fut extrait ałce difficulté; l'examen de cet œil, fait 250 jours (du 18 mai 1866 au 22 janvier 1S67) aprés l'opération, en dérnontra l'atrophice.

XI. I)ans la $40^{\circ}$ expéricnce à résultat positif, à un bélicr de 2 a 3 ans, il fut fait sur l'onil droil la léralolomic externe; l'cxamen le cet reil, fait 162 jours (fiu $1 / 1$ août 1866 au 22 janvier 1867) "pres l'opération, y dénontra la préscuce d'un cristallin régénéré de la grandeur d’un $1 /$ d du cristallin normal ; sa fornec élait ronde, 
mais aphatie a'alant en arriere; il élait constilue far des tuhes ct des fibres cristalliniens dont les uns ćtaicnt normaux et les autres grossis; la surfice interne de la cristalloüle antéricure élait z'couverte d'épithélium. Cette préparalion a été démontrée is l'Académie de médecine de Paris, dans la sciance du 29 janvier 1867 (royez pl. V, fig. 1).

Chats. - I)es / expériences faites sur des chals, 1 (12) cut un résullat positif, et 3 (11, /13 el \$4) un résultut négutif.

XLI. Dans la $h 1^{\circ}$ expérience à l'ésultal négatif, à un clat de 5 mois, il fut fait sur l'oxil gauche la liératotomie supérieure, l'exa-

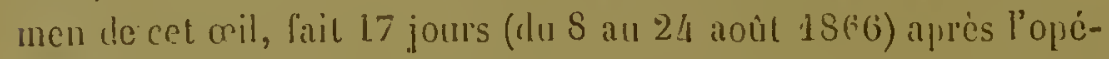
ration, démontra que la eristallö̈le antérieure, prés de sa partic lésée par l'aignille, était recouperte d'une membrane pignmenlée, el contenail du tissu conjonctif et des vaisscaux capillaires qui recouvraient une certaine partie de la surface antérieure de la eapsule cristallinicune. Celle derniere contenait du lissu conjonclif, el, par endroits, de l'épilluélium, des cellules formatrices, alignces par séries linćares, des tubes et fibres erislalliniens de différentes grindeurs et à bouts pyriformes et des globules de Morgagni (royez pl. V, fig. 2).

ALII. Wans li $42^{\circ}$ cxpéricnce à résultai positif, à un elat d'un an, il fut fait sur l'oeil droit la liératotomie supériezure; l'cxamen de ese oil, fuit 80 jours (dn 24 octobre 1860 au 11 janvier 1867) après l'opération, y démontra la présence d'un eristallin regcénéré dans sa moitie inlericure, et ayant la forme demi-lunalire; le hourrelet eristallinien élait très -peu accentué dans sa parlie supéricure; la fosselte annulaire étint ovale daus la direclion du diamelre horizontal de l'appareil cristallinien el reçowerte au milieu de pigment; le bourrelet demi-lunaire contenail des lubes el des fibres cristalliniens, et de l'épithélium; colle préparation a été démontrée à l'Académie de médecine de l'aris, le 29 janvicr 1867, el à la Scciété des médecins russes, de SaintPéterslourg, daus la sciance du $1^{\text {er }}$ avril 1868 (royez pl. VI, fig. 1, A, B).

XLIII. Dans la $43^{\mathrm{a}}$ expérienec à résultat mégatif, à un chal de 2 à 3 ans, il ful fait sur l'wil droit la kírutotomie supérienre; l'exa- 


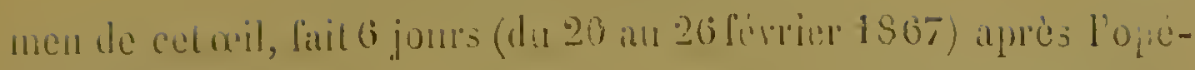
ration, dénondra une lésion T- lorme de la cristalloüde antérienre cl une lision longiludinale, de liaul ea las, de la cristalloüle posléricure; les deux cristallö̈des élaient trés-lapprochécs l’une de l'aulre, et la capsule contenait de l'épithélium, des tubes cristalliniens modiliés, des globules de Mor'grigni, des globules de šang, des cristaux d'liématoüdiue, des cellules du corps vilré de différentes formes et grandeurs el ayant subi la transformalion granulcuse, ct çà et lia de l'humeur villée (voyez pl. VI, fiğ. 2).

XLIV. Jans la $44^{\circ}$ expéricnce à résultás négatif, à un chat d'un an, il fut lait sur l'ail ganche la liératotomie supérieure; l'examen de cet oil, fait 129 jours (du 20 février au 28 juin 1867 ) aprèslopération, lémontra que les cristalloïdes adhóraient l'une à l'autre au moyen du lissu conjonclif, au sein duquel on trouvait, par' cndroits, de l'épithélium el des ccllules lormatrices; la partic anlérieure de la capsule élait couverte de lissu conjonclif.

Cochons d'Inde. - Des 3 expériences laites sur des cochons d'Inde, 2 (1 15 et 46 ) eurent un résultat positif, ct 1 (47) un résultat négatif.

XWV. Dans la $45^{c}$ expérience à résultat positif, à un cochon d'Inde de 3 mois, il fut fail sur l'cil gauche la liératotomie supérieure; l'iris fut blessé ct la capsule Guverte au moment de l'incision cornéenne; l'examen de cet cil, l'ait 77 jours (lu 18 aoùt au 2 norcmbre 1S66) après l'opération, y démontra la présence d'un cristallin régénéré annulaire avec une fosselte irréguliècment quadrangulaire ; la fossetle correspondait ì l'anneau, et adhérait à la partic supéricure de l'iris; l'anneau cristallinien contenait : de l'épithrilium, des lubes cristalliniens de différentes formes ct des globules de Morgagni (voyez pl. VI, fig. 3).

XLVI. Dans la $46^{\circ}$ cxpérience a résultat positif, a un cochon d'Inde de in mois, il fut fait sur l'wil droit la kératotomie supérienre, par suite de l'insulfisance de l'incision cornéen!e, le cristallin fut extrait aree dillicullo; l'examen de cetrril, lait 168 jours

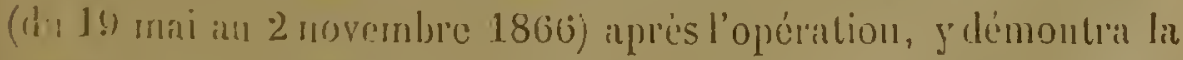
prisenee d'un cristallin regenéré et ayant la forme d'un fer it chevil; lit fossette annulatire avait mre forme irreguliere, et se 
continuait vers la pirlic interrompue de l'anneau, en liaul, ou elle adhérait à l'iris; la cavilé de l'anneau contenait : de l'épilliélium, des lubes et des libres cristalliuiens.

XLVII. Dans la $47^{\circ}$ expéricnce ì résultat négatif, it un coclion d'Inde de 2 mois, il fut fait sur l'wil droit la kératotomie externe; l'cxamén de cet oil, fait 118 jours (du 13 janvier au 10 mai 1867) après l'opération, en démontra l'atrophic.

Rats. - Des 2 expériences fuiles sur 2 rats (4S et 49), toules deux curent un résultat négatif.

XLVIII. Dans la $48^{e}$ expérience à résultat négatif, à un ral de 4 mois, il fut fait sur l'wil gauche la liératotomie supérieure; l'examen de eet oil, fait 118 jours (du 13 janvicr au 10 mai 1867) après l'opération, cn démontra l'atrophie.

XLIX. Dans la $49^{\circ}$ expérience à résultal négatif, à un rat de 4 mois, il futfait sur l'œil gauche la liératotomie supérieure; l'iris fut blessé, et le cristallin, par suile de l'insuffisance de la lésion corncenne, fut extrait avec difficulté ; l'examen de l'veil, fait 330 jours (du 20 janvier au 15 décembre 1867) après l'opération, démontra que la capsule adhérait par sa surface postérieure, à la réline, ct qu'clle ne contenait que quelques traces de tubes cristalliniens pàles et à buuts pyriformes.

\section{Conclusious.}

De toules ces expériences, ainsi que de celles des auteurs cités dans la partic historique de ce travail, il résulte :

$1^{\circ}$ Le fait incontcslable de la régénération, chez certains mamnifères, du cristallin, dont les liubes suivent dans leur réapparition les phases qu'ils offreut pendant leur génération el leur évolution embryonnaire.

$2^{\circ}$ Cette régénération n'a lieu que dans la cavité de la capsule cristallinicnne, elle est en raison directe de l'épaisseur des couches corticales du crislallin qu'on laisse dans la capsule, surtout dans sa partie équatoriale, pendant l'opération de l'extraclion; elle est en raisoninverse de l'àge des animaux et des lésions des erislalloïdes de la capsule eristallinienue. 
$3^{\circ}$ La régénération du cristallin a lieu ì la surface équatoriale interne de la capsule cristallinienne et à celle de la cristallö̈de antérieure. La cristalloïde postérieute ne scmble point prendre part à la régénération du cristallin; il faut en exempter cependant sa parlie équaloriale.

$4^{\circ}$ La régénération du cristallin a lieu non-seulement lorsqu'on laisse, pendant l'extraction du cristallin normal, une couche notable de sa substance corticale, mais encore lorsque le cristallin est extrait en tolalité. Si la quantité des couches restées n'est pas grande, ou bien si la caviti de la capsule cristallinienne ne se referme pas vite, les tubes cristalliniens qui y élaient restés sont r'ésorbés par l'huneur aqueuse. Ces lubes disparaissent par désagrégation.

$5^{\circ}$ La régénération du cristallin, Iorsqu'elle a lieu, ne devient appréciable à la vue cu'à parlir de la lin de la deuxième semaine après l'opération; elle n'est complète qu'entre le cinquième et le douzième mois, et nême plus tard, lorsque les animaux sont àgés. La régénération du cristallin est, par conséquent, une question de temps.

$6^{\circ}$ Les cristallins régénérés, obtenus jusqu'à présent dans nos sxpériences, ont atteint el mème dépassé de beaucoup la moilié du volume du cristallin normal, mais ils n'unt jamais atteint le volume du cristallin normal qu’ils ont remplacè. Cela est dû aux 'ésions de la cristalloüde antérieure el aux différentes complicalions de l'opération de l'extraction. Certains auteurs (Leroy d'Etiolles, Midlmore et Philipeaux), cependant, ont obtenu des cristallins régéućrés aussi grands ou presque aussi grands que les cristallins normaux.

$7^{\circ}$ Le cristallin régéncié a plus ou moins la forme, la densité et la transparence rlu cristallin normal extrait, et peut par consćquent, dirns les cas bien réussis, le remplacer au point de vue physiologique.

8" L'incision cristalloïdienue antéricure deni-circulaire, donnant un lambeau correspondant par sa lorme a celle de la cornće, is des conséquences capitales non-sculement sous le rapport de la regencration du cristallin, mais encore sous celui de la marehe 
des phénomènes consćcutifs à l'opération de l'exlraction du cris. tallin.

$9^{\circ}$ La régénération secondaire du cristalli!ı, c'est-á-dire celle qui a lieu après l'extraction d'un cristallin dejà régénéré unc fois, peut avoir lieu, mais elle est limitéc.

$10^{\circ}$ La structure microscopique des éléments du cristallin régénćré ne différe pas de ceux du cristallin normal; cependant il arrive très-souvent que les pronier's se modifient cl acquièrent un volume plus grand.

$11^{\circ}$ L'inflammation peu intense de l'iris et du corps ciliairc, loin de nuire à la regénération du crislallin, la favorise au contrairc. L'inllammation générale de l'œil (pauıophlıalmie) est un obstacle à la régénćration du cristallin.

$12^{\circ}$ Le lissu conjonctif de nouvelle formation trouvé dans quel. ques-unes de nos expériences dans la cavité de la capsule cristallinienne, etdont la production a été attribuée, à tort, par quelques autcurs aux éléments cristalliniens, est dù à l'épanchoment dans cette dernière soit du produit inflammatoire de l'iris enflammé, soit de l'humcur vitréc, après l'opération de l'extraction du eristallin.

\section{INDEX BIBLIOGRAPHIQUE.}

Ténon. Nlémoire et observation sur l'anatomie et la physiologie, etc. Paris, 1706, 1. I, p. 41 el 57.

A. Mattre Jean. Traité des maladies de l'wil. Troyes, 1707. Observ. IV, p. 116.

WinsLow. Exposition anatomique de la structur'e du corps humain. 1782, t. IV, $\S 233$.

A. HALLer. Elementa physiologiæ corporis humani. Auctore Alberto Haller. Lausanni, $1769, \mathrm{t} . \mathrm{V}, \S \mathrm{XXI}$, p. 469.

OLOF ACREL. Chirurgische Geschichte im kœniglichen Lazarelhe 7.1 Stockholm. Aus dem schwedischen. Lübeck u. Leipzig, 1772, p. 50.

- Chirurgische Forfälle in dem Königl. Lazaret u. auszerhalb demselben angemerkt. Gœuttingen, 1777, len Bd. p. 109, v. And. Mlurray.

ZINN. Descriptio anatomica oculi humani iconibus illustrata. Ciollingx, 1776. p. 138-142. 


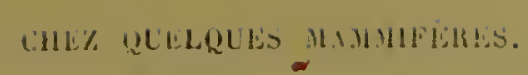

Peticunt l'otr. Chirurgical Works in thece volumes. Loudon, 1790, vol. 3, p. 237 .

E.IRL. Account of a new mode of operating c:alaract. 1801.

BuchNER. Waarneming van eene entbinding der Grystallvogten, elc. Ams. terdam, Is01.

T.ırtr.. De l'opélation de la cataracte (Thèse présentéc au concours). Paris, $1812, p .40$.

JaYER. Ueber Histologie und eine neue Eintheilung der Gewelse des mensclilichen Körpers. Bonn. 1819, p. 14.

SCARPA. Traité des principales maladies des yeux. Traduct. franç., 1821 , t. II, p. ‡ü.

FR. Dietericı. Ueber die Velwundungen des Linsensystemes. Tübingen mit 1 Steindrucktafel, IS24.

LERoY D'ÉTIOLLES. Expériences relatives à la reproduction du cristallin. Mćmoire lu à l'Acadèmie de chirurgie, le 10 févricr 1825.

- Journalde plrysiologie expérimentale el pathologique de Magendie. 1827 , t. VII, p. 30 .

- Recueil des lettres et mémoires adressés à l'Académie des sciences pendant les années de 1842-1843. Paris, 1844. Voy. aussi : In Froriep’s Notizen. no 3 \$9 vom Jahr 1827. 19 des XVI Bandes Sp. 289.

AD. HesSELBACH. Bemerliungen über die Operation des grauen Staares. Im IIten Bande der Beitræge zur Natur und Heilliunde. Herausgegeben, v. J. B. Friedreich u. A. Hesselbach. Nürnberg, 1826, p. 126.

P. BACkhatsex. De regeneratione lintis crystallina. Auctor Petrus Backliausen. Berolini, 1827 . Diss. inaug.

W. ScEnerisg. Beobachtungen über die organischen Veränderungan in duge nach Staaroperationen. Frankfurt, 1 S28.-Voy. aussi : In Journal hebdomadaire de médecine, 1828, t. I, p. 59-65: Observations sur les changements que l'œil éprouve après l'opération de la cataracte par abaissement.

H. Day. Reproduction of the lens in The Lancet, 1828 . November, P. 212.

R. Midssone. On the reproduction of the cristalline lens, in The London medicnl Gazelle, vol. X, 1832. June, 16, p. 344-348. Voy. aussi : Behrend's liepertor. Oct. 1831, p. 4 , etc.

VAver. Leber dic Reproduction der Krystallinse. Im Journal der Clirurgie und Augenlieilkunde v. Graefe u. Walther. Bd. XVIII. 1832. IVten Ileft. p. $\$ \$ 8-830$.

- Leber die lieproduction der kirystallinse von Prof. Mayer in Bonn. Im Journal der Cllirurgie und A:rgenlseilkunde von C. T. v. Graefe v. Ph. v. Walther. 1832. I3d. XYII, IIeft. IV, P. 521 et strirantes.

IIEschkE. Uehei die erste Entwickelung des Auges u. dic damil zusammenhängende Cyclopie. In Meclel's Archiv fir Anatomic und 'hysiologie. 1832. lid. V1. l.eiprig, 1. 16-20. 
BEGEr. De reactione traumatica iridis et anterioris capsulæ paritis. Lips., 1833.

k. Beck. De oculorum mutationilus qux cataraclæ operationem sequentur, observatio adnexis corolariis. Freiburg in Breisgau, 1833. Uehersetzt v. $D^{r}$ Beger in von Ammon's Zeilschrift fir die Ophthalmologie. $1 V^{\text {tea }} \mathrm{Bd}$. 1ten Ileft. 1835, p. 95-113.

W. Wenneck. "Einige Resultate meiner an verschiedenen Thieren gemachten Experimente über die traumatisclie Reaction hei Verwundungen der Kapsel und der Linse, nebst Angabe jener Erfahrungen üher diese Reaction beim Menschen. ) In der Zeitschrift für Ophthalmologie von prof. Ammon. 1835, IVten Bd., $1^{\text {ten }}$ Heft., p. 18-21.

J. Radius. "Cataracta. " In dem Handwörlerbuch der gesammten Chirurgie und Augenheilkuride herausgegeben von den Professoren W. Walther, II. Jäger und Radius. Leipzig, 1836. II Bd. II Lief, p. 302.

A. Retzıus. Fidskrift för Läkere. 1 837. Voy, aussi dans Schmidt's Jahrbücher' der in 1. ausl. ges. Mled. 6838. Bd. XlX.

Von F. A. Ammon. Klinische Darstellungen der líranliheiten des mensehlichen Auges. 1. Theil. Berlin. 1838.

PAuLl. Ueber den grauen Staar und die Verkrümmungen. Stuttgart, 1838 , p. 21-25. Voy. aussi : Annales d'oculistique et de gynécologie, publiées par F. Cunier et Scliönfeld. 1 vol. 24 livr. 1839.

Burdach. Trailé de physiologie, 1838, Trad. franç., t. vili, p. 288.

II. J. Ciselius. Handbuch der Augenheilkunde, v. Chelius. I Iten Bd. Stuttgart, 1839, p. $331-332$.

- Trailé pratique d'ophthalmologic, par Max. Jos. Chelius. Trad. par Ruef et Deiber, 4839, p. $314-315$.

LöwENHARDT. "Einige Versuche, um die Regeneration der Krystallinse zu documentiren. vIn Neue Nolizen u. s. w. von Froriep, no 418 (To 22 des XIXten Bandes), september 1841. Spalte 344-346.

K. TExtor. Ueher die Wiedererzeugung der Krystallinse. lnaugural Abhandlung v. Karl Textor aus llünchen. Mit 3 Steindrucktafeln. Würzburg, 1842.

Hanmans. Ueber reproductio lentis, von $D^{r}$ Hanmann, zu Rostock. Im llirfland's Journal des Praklischen Heilkunde. 184z, p. 102.

HENLE. Traité d'anatomie générale. Trad. franç. 18\$3, p. 368 .

VALENTIN. Mikroscopische Unter'suchung zwoier wiedererzengten Iírystalllinsen des Kaninchens v. Valentin. lin Henle u. Pfenfer. Zeitschrift f. ration. Medicin. 184\%. Iten Bd. $2^{\text {ton }}$ partie. Voy; aussi. C. Clır. Schmidt's Jalırbïclier d. in u. ausl. gesammten Nediciu. Jahrg. 18\% 4 . XLIIten Bd. $1^{\circ} 2$.

WaLker. Uıter'suchung eines A uges 6 Jahr' naclı Auszielıung der Linse. Iı C. Clır. Schmid's Jahrls. d. Gesammt. Mled. 1845. XLVIIIten Bd. p. 215.

1. Broca. Présentation de dleux yeux d'un liomine de 60 ans, mort avec deux cataractes lenticulaires. In Bulletin de la Société analomique de Paris, $22^{\circ}$ année $(1847)$, p. $435^{\circ}$. 
Nerer. Beitrag zu der Streitfrage über die Enstehung der Linsenfasern. Im Müller's Archiv f. anatomie u. physiologie. $183 \%$, p. 201.

Follwx. Examen d'un œil opéré de la cataracte par extraction quinze ans avant la mort du malade. In Annales d'oculistique. Paris, $185 \% 1$ t. XXV, $f^{\text {er }}$ sem.

Sichel. Iconograplie ophthalmologique. Paris, 1852-1859, p. 263-273.

Desvarres. Traité théorique et pratique des maladies des yeux. Paris, 1858 , $z^{e}$ éd., t. III, p. 386 .

D’Avaros. Ophthalmogénèse. In Annales d'oculistique. 1859, t. XLII, $8^{\mathrm{e}}$ série, p. $179-200$.

Richet. Traité pratique d'anatomie medico-chirurgicale. Paris, 1860, p. 350 , et $1871,4^{\circ}$ éd., $2^{\mathrm{e}}$ partie, p. 118.

F. J. vos Becker. Untersuchungen ueber den Bau der Linse bei dem Menschen u. den Wirbelthieren. Im Archiv für Ophthalmologie v. Arlt, Donders u v. Graefe, 1863 , Bd. IX. IIten $\Lambda$ bth.

BaBouchıN. Beiträge zur Entwickelungsgeschichte des Auges. In Würzb. Yalurvissensch. Zeilschrifl. 1864.

Hrrtl. Handbuch d. topographischen Anatomie. Iten Bd. 1865, P. 232.

Ross. Handbuch der chirurgischen Anatomie. Année?...

ZERxow. Zum mikcroscopischen Bau der Linse beim Menschen $u$. bei den

Wirbelthieren. v. Zernolf in Moskau. In Archiv \%. Ophthalmologie, v.

Arlı, Donders u.v. Graefe. 1867, Bd. XIll. Abth. II, p. 521-546.

Зерновљ, 0 mикроскопичскомь строеніи хрусталика у человька и позвоночныхъ животныхъ. Ансесртація. Москва, 1867.

(ZERiow. De la structure microscopique du cristallin chez l'homme et les vertébrés. Moscou, 1868. Thèse inaug.)

B. Miluot. Mémoire sur la régénération du cristallin. In Comples rendus de $l$ Academie des sciences. 28 janvier 1867.

В. Милдіотљ, Опыть возрожіденія порлальнаго хрусталика у ићкоторыхъ ц.непитаюцихъ животныхъ, посль удаленія его .оскутныъ сыченіемь. Аиссертація. С. Петербургь, 1868.

(B. Mlluot. Essai sur la reproduction du cristallin cliez quelques mammiféres aprẻs l'extraction du cristallin normal. Saint-Pétersbourg, 1868. These inaug.)

L. WECKER. Traité théorique et pratique des ınaladies des yeux. Paris, 1868 , $2^{*}$ édit., t. II, p. 12.

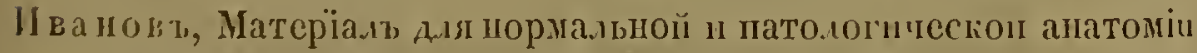
хјустал пlia. Аиесертаціл. С. ІІетербург"ы, 1867.

(IVA.sow. Matériaux pour servir à l'anatomie normale el palhologique du cristallin. These inaug., Saint-Pélershourg, 1868.)

IVAsiow. Beiträrge \%ur normalen und pallologischen Anatomie des Auges. In Archiv fur Ophthalmologie, llerausg. v. Arlt, Dondess u, v. Gracte XVtun Jahrg. Abtl. 1J. 1869, 1). 1-107.

J. Phurfaux. Expériences montrant que le cristallin peut se régérérer clıez 
les mammifères par une formation nouvelle dans la capsule cristallinienne. In Gazetle médicale. $1870, \mathrm{n}^{\circ} 46, \mathrm{p} .577$, Sénnce du 23 avril 1870 de la Société de Biologie.

\section{EXPIICATION DES PLANCIIES 1, II, III, IV, $\mathrm{V}$ et VI. \\ Planche I.}

f'ig. 1. liystitomes employés par l'auteur pour ouvrir lat capsule cristallirienne.

1. Kystitome de Cheselden avec cuilles de Daviel.

b. Kystitome genouillé (voy. pp. 29 et 36 ).

FIG. 2. Appareil cristallinien trouvé, dans l'nil droit d'un lapin, cent quarante-sept jours après l'opération de l'extraction (expérience Xl11e). $a, b$. Fibres cristalliniennes.

c. Tissu conjonctif de nouvelle formation trouvé dans l'anneau près de de la fossette annulaire.

1. Anneau cristallinien avec sa fossette irrégulièrement ovale.

(Oberhäuser, oculaire 3, objectif 7.)

Fig. 3 et 4. Cristallins normal et régénérés obtenus j'ar Philipeaux et déposés alu musée Dupuytren (Maladies des yeux, n 11 ). Le cristallin normal est présenté de face et de profil dans le but d'expliquer la nomenclature de l'appareil cristallinien (voy. p. 4).

FIG. 3. A. Cristallin normal de lapin vu rle face et grossi une fois et demie. $a, b, c, d$. Equateur de l'appareil cristallinien.

n,b. Diamètre vertical.

$c, d$. Dianètre horizontal.

B. Même cristallin vu de profil.

$c, f$. Axe de l'appareil cristallinien.

¿. Pôle postérieur.

$f$. Pôle antérieur.

l'li. 4. Cristallins régénérés de lapin, grossis une fois et demie.

$a^{\prime}$. Anneau cristallinien avec sa fossette, trouvé quinze jours après l'opération de l'extraction du cristallin normal.

l'. Bourrelet cristallinien, occupant la partie inférieure de l'appareil cristallinien, trouvé vingt-cinq jours après l'opération.

$a !$ Anneau cristallinien très-accentuć, et sa fossette annulaire irrégulière, trouvés quarante jours après l'opération.

$d^{\prime}$. Cristallin régẻnéré, trouvé soixante jours après l'opération et ayant itteint les trois quarts du cristallin normal; la fossctte annulaire est très-petite.

$e^{\prime}$. Cristallin régénéré, trouví cent vingt-deux jours (quatre mois) après l'opération. 
$f^{\prime \prime}$. Cristallin régénéré, trouvé cent cinquante-trois jours (cinq mois) après l'opération, et ayant presque atteint la forme et la dimensien du cristallin normal.

Fı. วั. Appareil cristallinien trouvé, chez un lapin, soixante-treize jours après l'extraction du cristallin normal (expérience $\mathrm{X}^{\mathrm{ro}} \mathrm{V}^{\mathrm{O}}$.

ı. Tubes cristalliniens.

๖. Tubes cristalliniens et zone nucléaire (Kernzone) de Meyer.

c. Cellules épithéliales de la capsule cristallinienne.

c. Cristallin régénérè, formé par deux bourrelets, dont l'un, l'inférieur, est grand et bombé, et le supérieur est mince ; la fossetle annulaire a la forme oblongue et est transparente.

- (Oberhäuser, ocul. 3, obj. 7.)

\section{Planche II.}

Fli. 1. Cristallin régénéré trouvé, chez un lapin, quarante-six jours après l'opèration de l'extraction du cristallin normal (expérience XVIII ${ }^{\mathrm{e}}$ ).

a. Cristallin formé par deux bourrelets demi-lunaires : le supérieur plus petit et l'inférieur plus grand ; la fossette annulaire est transparente et a l'aspect d'une fente.

$b$. Tubes cristalliniens plus grands qu'à l'ètat normal, avec des bouts grossis et pyriformes et un grand globule de Morgagni.

$c, d$. Tubes cristalliniens plus grands qu'i l'état normal et présentant des noyaux hypertropliés.

$e$. Tuhes cristalliniens de grandeur normale.

$f, f, f$. Fibres cristalliniennes.

(Nachet, ocul. 2, obj. 3.)

Fig. 2. Cristallin régénèré d'un chien, trouvé quarante-deux jours après l'extraction du cristallin normal (expérience XXIle).

1. Cristallin formé par un bourrelet en fer à cheval avec deux stries allant de la fossette ovale et transparente à l'équateur de la capsule cristallinienne.

1. Épitlélium de la cristalloüde antérieure.

c. Préparation dans laquelle on voit la disposition des cellules épithéliales, des cellules de Robin et des cellules formatrices de Becker.

c. Épithélium cylindrique de la partie équatoriale de la capsule cristallinienne.

d. Couche des cellules de Rohin faiblement dessinée.

e. Cellules formatrices de Becker', parallèles à la surface capsulaire.

$f, f$. Tubes cristalliniens.

4,\%. Filires cristalliniennes. (Nachel, ocul. 3, obj. 3.)

Planche $1 \mathrm{II}$.

Fic, 1. Apparcil cristallinien trouvé, che\% un chien, cinquante et un jours apres l'operation de l'exiraction du cristallin normal (exprérience XXIII"). 
a. Tissu conjonctif de nouvelle formation recouvrant la fossette annulaire.

b. Bords de l'endroit de la cristalloïde antérieure, lésée par le kystitome, et adhérant les uns aux autres au moyen d'une substance amorplie.

c. Globules de Morgagni et bouts de tubes cristalliniens modifiés.

d. Cellules épithéliales.

e. Globule de Morgagni isolé.

$f, f$. Cellules fusiformes.

(Nachet, ocul. 3, obj. 5.)

Fig. 2. Un morceau de la cristalloïde antérieure, trouvé chez un clien quatre jours après l'opération. Ce morceau était recouvert, à sa surface interne, d'une substance finement granuleuse, d'un vaisseau capillaire, de quelques cellules formatrices de Becker, et de tubes cristalliniens, l'un pyriforme, les autres en voie de transformation granuleuse; au-dessus du dessiı, et à droite, on voit quatre globules pyoïdes (expérience XXIV ${ }^{\circ}$ ).

Fıg. 3. Appareil cristallinien trouvé clıez un vieux chien, cent quarantequatre jours après l'opération de l'extraction du cristallin normal (expérience XXVIII ${ }^{e}$.

a. Anneau cristallinien avec sa fossette triangulaire et transparente. A droite, on voit deux stries allant de la base de cette dernière à l'équateur de la capsule cristallinienne.

b. Épithélium de la capsule.

c. Cellules de Robin, qui, les ayant, le premier, distinguées des globules de Morgagni, les a, à tort, appelées cellules de Morgagni (royez p. 10$)$.

d. Quelques cellules formatrices de Becker éparses.

e. Fibres cristalliniennes normales en voie de transformation granuleuse.

(Nachet, ocul. 3, obj. 5.)

Fig. 4. Appareil cristallinien trouvé, chez un chien, neuf jours après l'opération de l'extraction du cristallin normal (expérience $X_{X I X}^{c}$ ).

a. Surface antérieure de l'appareil cristallinien grossi, sur laquelle on voit les bords recroquevillés de l'endroit lésé de la cristalloìde antẻrieure.

(Nacliet, ocul. 1, obj. 1.)

b. Surface postérieure de la même préparation de grandeur naturelle. A travers la cristalloĭde postérieure saine on voit les bords de la cristalloïde antérieure.

c. Une partie de la surface interne de la cristalloïde postérieure recouverte, près de l'équateur, de cellules de Robin.

d. Une partie de la cristalloïde antérieure (surface interne) recouverte d'épitlélium, de cellules formatrices de Beclier disposées d'abord parallèlement, ensuite perperdiculaireunent à cette même cristalloïde. 
$\therefore$ Cellules fusiformes.

f. Cellules formatrices le Becker éparses.

g. Deux bords de la cristalloïde antérieure adhérant l'un à l'autre au moyen du tissu conjonctif de nouvelle formation. A la partie inférieure ilu dessin, on roit des tubes cristalliniens en voie de transformation granuleuse.

(Nachet, ocul. 3, obj. 3.)

\section{Planche IV.}

Fig. 1. Appareil cristallinien trouvé, chez un chien, cent vingt-six jours après l'opération de l'extraction du cristallin normal (expérience XXX' $^{\mathrm{t}}$ ).

a. Capsule cristallinienne divisée en deux moitiés inégales.

b. Yoitié externe de la capsule contenant des fibres cristalliniennes non modifiées, de l'épithélium, des grains et granules pigmentaires et des cristaux d'hématoïdine.

(Nachet, ocul. 3, obj. 3.)

Fig. 2. Une partie de l'appareil cristallinien trouvé, cliez un chien, huit jours après l'opération de l'extraction du cristallin normal (expérience XXXIII $)$.

a. Un morceau d'une partie équatoriale de la capsule cristallinienne.

b. Corps ciliaire.

c. Zonule de Zinn.

Les cellules formatrices de Becker sont disposées d'abord parallèlement et ensuite perpendiculairement à la capsule cristallinienne.

d. Une partie de la surface interne de la cristalloïde antérieure, détachée à l'endroit où celle-ci avait été lésée par l'aiguille, et recouverte de quelques cellules formatrices, de grains pigmentaires et de cellules granuleuses de l'humeur vitrée.

(Nachet, ocul. 3, obj. 3.)

FIs. 3. Les cristallins normal et régénéré trouvés, chez un bélier, trentesept jour's après l'opération de l'extraction du cristallin normal et ayant subi longtemps l'influence de la solution de bichromate de potasse (expérience XXXVII $\left.{ }^{\mathrm{e}}\right)$.

FIG. 3. A. Eléments du cristallin normal.

a. Noitié du cristallin normal coupé en deux pour montrer sa forme, sa grandeur naturelle et son noyau fortement accentué.

৮. Une couche de tubes cristalliniens et de cellules de Robin détachées de la cristalloỉde antérieure.

$c$ et $d$. Fibres eristalliniennes.

\%. Espace interfibrillaire.

li. Éléments du cristallin régénéré.

a'. Woitié du cristallin régénéré, coupé en deux pour montrersa forme, sa grandeur naturelle et son noyau, moins accentué que dans le cristallin normal.

u'. Morceau de la partie périphérique du cristallin régénéré, détaché 
de la capsulc. On y voit les bouts de tuljes cristalliniens se terminant derrière unc couclıe granulcuse (produite par la solution de bichromate de potasse), dans laquelle on distingue avec peine des cellules épithélialcs; au-dessus de cctte couche on voit (à droite) les cellules dc Robin et les noyaux des tubes.

$c^{\prime}, d^{\prime}$. Terminaison des tubes cristalliniens plus larges qu'à l'ćtat normal.

$e^{\prime}$. Espace interfibrillaire un peu plus grand qu'à l'état norınal et rempli de globules ronds et dont le contour se dessine à peine.

(Oberhäuser, ocul. 3, obj. 8.)

\section{Planche V.}

Fıg. 1. Tubes et libres cristalliniens des cristallins normal et régénéré, trouvés, cliez un bélier, cent soixante-deux jours après l'opération (expérience $\left.X L^{e}\right)$.

Fic. 1. A. Éléments du cristallin normal.

a. Tubes cristalliniens.

$b, c, l, e$. Fibres cristalliniennes.

B. Éléments du cristalliı régénéré

$a^{\prime}, b^{\prime}$. Fibres cristalliniennes.

$c^{\prime}, d^{\prime}$. Tubes cristalliniens.

(Nacliet, ocul. 3, obj. 3.)

Fıg. 2. Appareil cristallinien trouvé, cliez un chat, dix-sept jours après l'opération de l'extraction du cristallin normal (expérience $\mathrm{XLI}^{\mathrm{e}}$ ).

a. Cellules pigmentaires éparses trouvées dans la partie de l'anneau cristallinien, avoisinant l'cndroit de la cristalloïde antérieure, lésé par le kystitome.

b. Cellules formatrices de Becker alignécs par séries linéaires.

c. Bords de la cristalloïde antérieure de l'endroit lésć par le kystitome et adhérant l'un à l'autre au moyen du tissu conjonctif de nouvelle formation.

d. Surface antéricure d'une partie de la cristalloïde antérieure recouverte de tissu conjonctif dc nouvelle formation et de vaisseaux car illaires; derrière la partie transparentc de la cristalloïde on apcrçoit quclques cellules formatrices de Bccker éparses.

e. Bouts de tubcs cristallinicns pyriformes el globules de llorgagni.

$f$. Fibres cristalliniennes.

(Nachet, ocul. 3, obj. 5.)

\section{Pianciie Vl.}

FIG. 1. Cristallins normal et régénéré trouvés, cliez un cliat, quatre-vingts jours après l'opération de l'extraction dn cristallin normal (expérience $\mathrm{XI}^{\mathrm{I}} \mathrm{I}^{\mathrm{e}}$ ). 
Fis. 1. A. Eiléments du cristallin normal.

a. Cristallin normal.

b,c. Fibres cristallinienues.

B. Éléments du cristallin régénéré.

$u^{\prime}$. Cristallin régénéré constitué par un grand bourrelet demi-lunaire occupant la partie infërieure de la capsule cristallinienne, et par un petit bourrelet étroit situé à la partie supérieure de la même capsule; au milieu et près du pôle antérieur de l'appareil cristallinien on voit un dépồ de pigment.

$U^{\prime}$. Épithélium ayant subi longtemps l'action du mélange de Legros (glycérine, arsenic, solution de colle et camplure); aut-dessous on voit des fibres cristalliniennes.

$c^{\prime}$. Tubes cristalliniens plus grands qu'a l'état normal.

a. Tubes cristalliniens normaux.

e. Une partie de la capsule cristallinienne et cellules formatrices de Becker ayant subi l'action du mélange de Legros.

(Oberhäuser, ocul. 3, obj. 8.)

FIG. 2. Appareil cristallinien trouvé, chez un chat, six jours après l'opération de l'extraction du cristallin normal. L'incision de la cristalloïde antérieure a la forme de $T$, et celle de la cristalloïde postérieure est longitudinale (expérience XLIII ${ }^{e}$ ).

a. Cellules épithéliales cylindriques, tubes cristalliniens modifiés et à bouts pyriformes, globules de Jorgagni et grains et cellules pigmentaires.

$b, b$. Cellules granuleuses du corps vitré.

$c, c$. Globules du sang.

d. Cellules épithéliales.

e. Cristaux d'hématoïdine.

(Nachet, ocul. 3, oljj. 3.)

Fig. 3. Cristallin régénéré trouvé, chez un cochon d'lnde, soixante-dix-sept jours aprés l'opération de l'extraction du cristallin normal (expérience XLVe).

a. Forme en fer à cheval et grandeur naturelle du cristallin régénéré. La fossette annulaire est très-accentuée.

b. Tulses cristalliniens.

$c, e, f$. Fibres cristalliniennes.

d. Cellules épithéliales.

y. Cilobules de Morgagni.

(Naclict, ocul. 3, obj. 3.) 


\section{COMPOSITION DES CALCULS BILIAIRES HUMAINS}

\section{Par M. E. RITTEIR,}

Agrégé à l’ancienne Faculté de médecine de Slrashourg.

Nos connaissances sur la composition des calculs bilinires sont loin d'être aussi avancées que celles que nous possédons pour les caiculs qui se forment dans le rein ou la vessic. C'est cetle lacune que j’ai essayé de combler, par les recherches suivantes, qui présenteront, je le pensc, non-sculement de l'intéret pour le physiologiste, mais qui intéresseront également les médecins praticiens. Mes analyses ont porté sur un nombre de concrétions très-considérable; le désir que j'avais d'obtenir une certaine quantité de cholestérine m’avait engagé depuis plusieurs années à ne rien négliger pour rćunir autant de calculs que possible. Des amis obligeants m'ont facilité cette tâche au point que je possédais près de 6000 calculs, au moment de commencer mes analyses.

En ne considérant d'abord que le poids des concrétions biliaires, j'en ai trouvé :

\begin{tabular}{|c|c|c|c|c|}
\hline 3920 & aien & de. . . . . & $\begin{array}{l}\mathrm{Gr} \\
0,1\end{array}$ & \\
\hline 108 & - & $\ldots \ldots$ & 0,1 i & 0,5 \\
\hline 160 & - & $\ldots \ldots$ & $0,5 \mathrm{a}$ & 1,0 \\
\hline 270 & - & $\ldots$ & 1,0 à & 2,0 \\
\hline 230 & - & $\ldots \ldots$ & 4,0 & 6,0 \\
\hline 22 & - & $\ldots \ldots$ & $6,0:$ & 10,0 \\
\hline 9 & - & $\ldots \ldots$ & 10,0 & 12,0 \\
\hline 3 & - & $\ldots \ldots$ & 12,0 i & 14,0 \\
\hline
\end{tabular}

Je ferai remarquer que je ne parle ici que des calculs qui ont été recueillis sans triagc préalable; on a mis de còté tous les calculs qui se présentaient à l'autopsic, qu'ils fussent grands ou petits, blancs ou colorés ; j’ai laissć de còlé dans ce tableau tons les échantillons qui me furent adressés et qui avaient été choisis. 
Je ne possède pas autant de données sur le uombre des calculs qui se trouvilient dans chaque vésicule; quelques-uns de mes collecteurs n'avaient malheureusement pas tenu comple de mes recommandations à cet égard. Voici cependant 75 observations recueillies dans de bonnes conditions.

$$
\begin{aligned}
& 7 \text { cas, } 1 \text { calcul. - Poids maximum, } 6 \text { grammes; minimum, } 2 . \\
& 3-2-\quad \text { Poids moyen, } 4 \text { gr }, 5 \text {. } \\
& 9-3-\quad \text { Les calculs qui se trouvaient dans chaque vésicule étaient } \\
& \begin{array}{l}
\text { presque égaux; } \\
1-4-\quad \text { Poids de } 1 \mathrm{gr}, 5 \text { à } 2 \text {. }
\end{array} \\
& 2-6 \text { Les calculs presque ćgaux entre eux pesaient chacun } 2 \mathrm{gram} \text {. }
\end{aligned}
$$

Les calculs présentent à la coupe des différences d'aspect très. notables qui permettent de les classer en plusieurs catégories : nous allons voir à l'instant que ces.différences répondent à des. différences chimiques dans leur composition. J'ai laissé de côté les calculs qui pesaient moins que $0^{\text {gr }}, 1$; ils avaieut la forme tétrnédrique le plus souvent. Les autres calculs peuvent ètre divisés en 8 classes.

$1^{\text {re }}$ Classe. Nombre des calculs, 28.

Ces calculs blancs ou jaunes cireux ont la forme ovoïde ou sphérique, l'aspect extéricur bosselé ; quelques-uns sont translucides, d'autres sont opaques. Leur poids est très-notable; ils n'ont jaınais pesé moins que $2^{\mathrm{gr}}$, le plus grand pesait $9^{\mathrm{gr}}$, et on m'en a mème remis un depuis qui pése 28 grammes. A la coupe on rumarque lat mème leinte qu'a l'extérieur; ils sont formés de cholestérine presprue pure, leur cassure est nettement cristalline; les cristaux rayonnert d'uu centre.

2: Ciasse. Nombre de calculs, 16.

Mème cuileur que les précédents, mème forme, que l'on ne 
peut rapporter qu'i celle d'un tonncau; ils présentent sur les parlies planes des enfonccments; leur contour est lisse, loujours opaque, quelquefois colori superficiellement par plaques. Leur eassure est eireuse et la coupe donne une couclic égale, ne présentant pas de traees de cristallisation.

$3^{\text {c }}$ Classe. Nonilire de ealculs, 580 .

Leur couleur est très-variable ; il en esl de mème de lcur forme, on en rencontre d'ovoïdes, de sphériques, de tétraédriques, etc. A la coupe on reconnait un mélange intime de eholestérinę et de malière colorante, mais la elolestérine a l'aspeet pailleté et les cristaux semblent r'ayonner du centre.

$4{ }^{\circ}$ Classe. Nombre de ealeuls, 94 .

Ces eonerétions ne diffèrent des préeédentes que par leur slructurc amorphe, ils ont très-fréquemment la forme d'un tonucau el se rencontrent rarement seuls dans un vésicule.

$5^{\text {e }}$ Classe. Nombre de ealculs, 220.

Ies ealculs présentent l'aspect cxlérieur des calculs opraques de la première classe, mais à la eoupe on voil des couehes alternantes et bien distinetes de cholestćrine amorplie el de inatière eolorante.

$6^{\mathrm{e}}$ Classe. Nombre de ealculs, 16.

Celte elasse ne différe de la précédente que parce que la couche cxléricure est forméc par de la matière colorante.

$7^{c}$ Classe. Nombre de caleuls, 3 .

Ces caleuls ne renferment que des traces de cholesterinc; deux trouvés dans la même vésicule pesaient $0^{\text {gr }}, 8$ et 0,7 , n'élaient formés que de matière colorante; on y voyail des zones d'un rouge vermillon, qui élaient de la bilirubine presque pure. Le troisième caleul, d'un brun foneé, était prineipalement formé par des mueus et de la bilihumine; il se laissait ceraser entre les doigls.

$8^{\mathrm{c}}$ Classe. Nombre de caleuls, 1.

Caleul dur, pesant, ne se laissant pas rayer par l'ongle; strueture amorplic, couleur gris sale.

L'ispect de la coupe peut encore nous donncr des renseignements sur la nature du noyau ou centre de cristallisation des cal- 


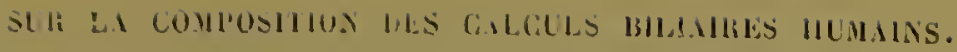

culs, ce qui permel d'aroir quelques notions sul les conditions qui ont présidé à la formation des dépóts.

29 fois, le noyau étail forué par de la cholestérine blanche;

650 fois, par un petit noyau de matière colorante, qui souvent b'élail visible qu'à la loupe.

267 fois, je n'ai pu distingucr nettement de hoyau el de point central.

Ajoutons enfin, pour lermincr les considérations que le simple aspect des coupes nous fournit, que les calculs avaient une structure uniforme dans 722 cas, qu'ils présentaient deux couches distinctes dans 208 cas, trois dans 24 et quatre dans 2 cas.

L'analýse chimique des calculs a été conduite de la manière suivontc. Au lieu d'analyser chaque calcul, j’ai opéré sur un nombre de calculs plus considérable en choisissant ceux de la mème classe. La poudre homogène a été desséchée ì la température de $+105^{\circ}$; un certain poids de cette poudre fut traité à froid par de l'éther absolu jusqu'à épuisement complet; les solutions éthérées, évaporées à siccité, ont laissé un résidu qui, jesé après dessiccation à $+105^{\circ}$, représente la cbolestérine des analyses qui suivent. Les sels ont été déterminés par la calcination; la différence entre les deux pesées et le poids tolal employé représente les matières organiques, sur la composition desquelles je reviendrai plus loin. Notons ici que le chiffre qui, d'après quelyues auteurs (Gerhard, Dictionnaire de chimie), indique la solubilité de la cholestérine dans l'éther, est erroné; ce n'est pas 3,7 d'éther qu'il faut à $+15^{\circ}$ pour dissoudre 1 de cholestérine, mais bien 6,7 .

Lcs résultats des analyses figurent dans le tableau suivant :

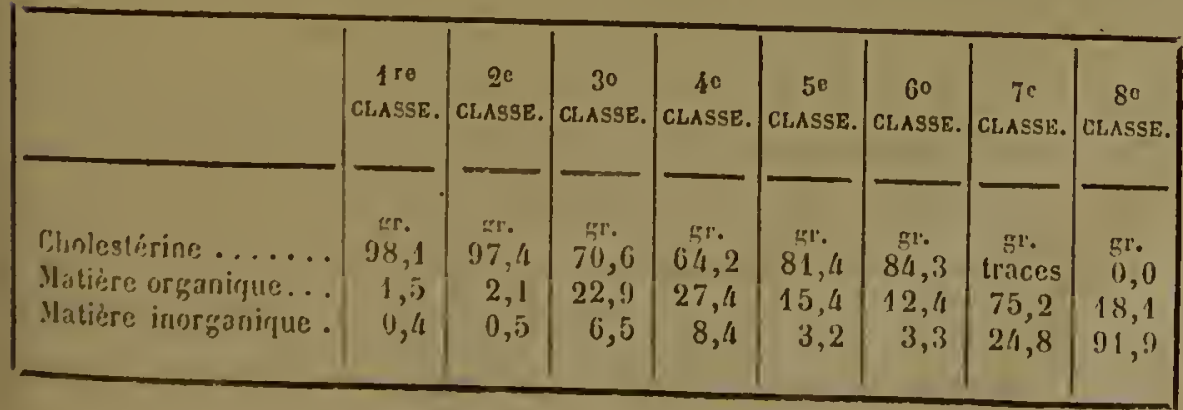


Un premier fait à noter cst la corrélation qui existe entre le poids de la matic̀re organique et de la matière inorganique; une augmentation de l'une correspond à ure angmentation de l'autre; le fait s'explique aisément, car nous allons voir bientot que les matières organiques sont à l'état de composćs calciques.

Je me suis demandé si le corps quc j’avais ainsi isolé par l'éther étuit de la cholestérine pure, ou s'il n'était pas formé par un mćlange de cholestérine et d'un corps gras. la cholcslérine isolée présentait le point de fusion de la cholestcrine pure, mais je ne me suis pas contenté de cette indication. J'ai fondu lc produit isolé par l'éther avec de la potasse au creuset d'argent, et j'ai repris la masse refroidie par de l'eau; la cholestćrine ne se saponilie pas dans ces conditions, ne se dissout que faibicnicnt dans le liquide alcalin; les corps gras au contraire auraient formé dcs savons potassiques solubles par ce mode de traitement. En traitant les savous solubles par un acide faible, on voit se séparer les acides gras insolubles; ma cholestćrine, traitée comme je viens de le dire, ne donnait jamais qu'une légère couche, preuve de l'absence de corps gras en quantité pondérable. Une seule fois il s'est produit un précipité qui correspondait à $1^{\mathrm{gr}}, 7$ de corps gras, compté comme margarine.

Lcs malières organiques contenues dans les calculs sont de nature trés-diverse; j'y ai décelé des inatières colorantes à l'état de composés calciques, des sels d'acides gras, du mucus. Je relate ici une analyse détaillée des calculs de la $3^{\circ}$ et $4^{\mathrm{e}}$ classe, qui sont les plus riches en ces composés et que j’ai utilisés avec beaucoup d'avantage pour l'extraction des matières colorantes biliaires. L'analyse a été faite d'après la méthode de Hoppe.Seiler (Bulletin de la Société chimique, 1869).

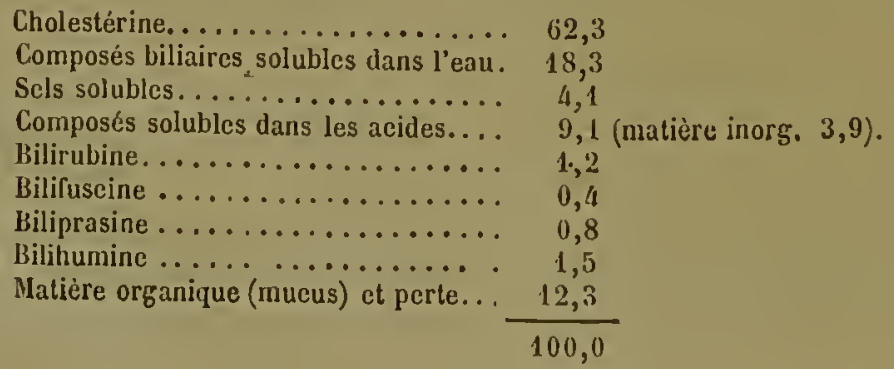


On rencontre parfois des calculs qui sont encore plus riches en matière colorante; je citerai conme exemple l'analyse de calculs de la $7^{e}$ classe, où la bilirubine était assez abondante pour former à la coupe des stries d'un vermillon très-vif.

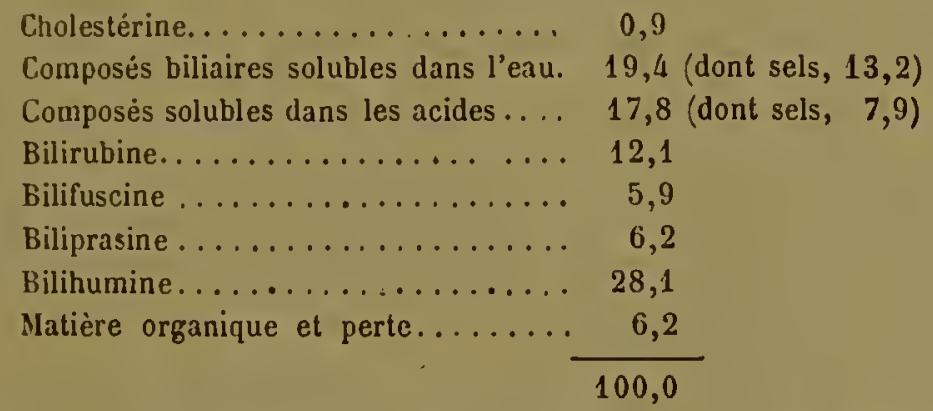

Les calculś dans lesquels dominen l les substances inorganiques sont une rareté; le calcul de la $\dot{\delta}^{\circ}$ classe a été retiré directement par moi de la vésicule d'une vieille femme morte à la suite de pneumonie; je n'ai pas réussi à ohtenir des renseignements précis sur ses antécédents. L'analyse délaillée du calcul est la suivante. Je n'ai pas dans les auteurs trouvé de cas semblable. Le calcul pesail 1,36 .

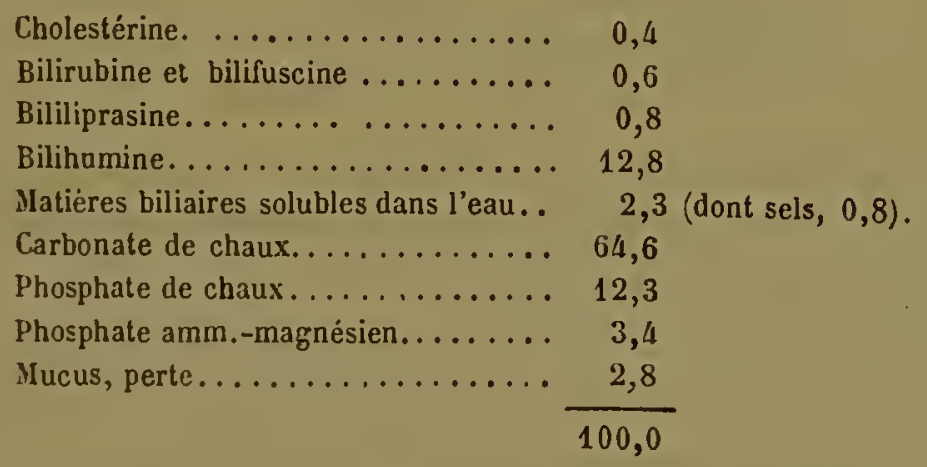

J'ai déterminé également la composition chimique des matières inorganiques laissées par les calculs des diverses classes. Les cendres ramenaienl loujours au bleu le papier de tournesol rouge; comme d'autre part les calculs ue produisaient sous l'influence des acides qu'un dégagement de gaz carbonique trèsfaible, nullement en l'apport avec l'acide carbonique qu'aurait exigé l'analyse alcalimétrique des cendıes, il fallait en conclure que l'alcalinite; des cendres ne pouvail provenir uniquement de 
la decomposition seule des chrlonales terrenx. Ce sont des combinaisons organiques de chaux qui, par leur decomposition pár la chalenr, produisent en majerre partic l'alcalinité du résidu. Des recherches faites sur des calculs avant l'incinération mont fait voir que l'oxalate de chaux n'existait pas dans ces concrétions, en quantité notable. Ce sont done les pigments et les acides biliaires qui sont combines à la chaux, et qui sous l'influence de la chaleur laissent un résidu de chaux vive. Les phosphates et les sullates ne se trouvent qu'eı quantité très-faible dans ces cendres, et encore n'est-il pas sûr que ces derniers ne proviennent pas de la décomposition des taurocholates.

Je ne relate ici que le résultat des analyses des calculs de la $3^{\circ}$ et de la $4^{\circ}$ classe; je lerai remarquer que la chaux qui figure à l'état de carbonate primitif a eté calculéc d'après la quantité d'acide carbonique dégagé par un poids donné de calculs traité dans un pelit appareil á poids de Bobière.

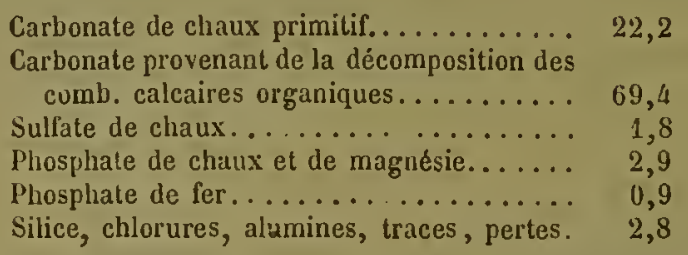

Je n'ai pas voulu borner mes analyses à cette étude d'ensemble; d'autres questions, qui pouvaient être résolues par l'analyse chimique se posaient naturellement.

Les calculs contenus dans la mème vesicule ont-ils une composition chimique identique ou différente? Il m'a semblé que les résultats obtenus pourraient permettre de tirer quelques conclusions relatives à l'époque de formation de ces concrétions. Se forment-elles sinultanénent ou à des moments dilférents? Les analyses ont porté sur 22 cas; toujours les calculs pesaient plus que 1 gramme ; 17 fois les différences étaient si légères, qu'on pouvait les regarder comme provenant des erreurs de l'analyse; cinq fois au contraire les diffírences étaient plus prononcées; jc ne contente de relater ici les truis analyses les plus concluantes à ce point de vue. 


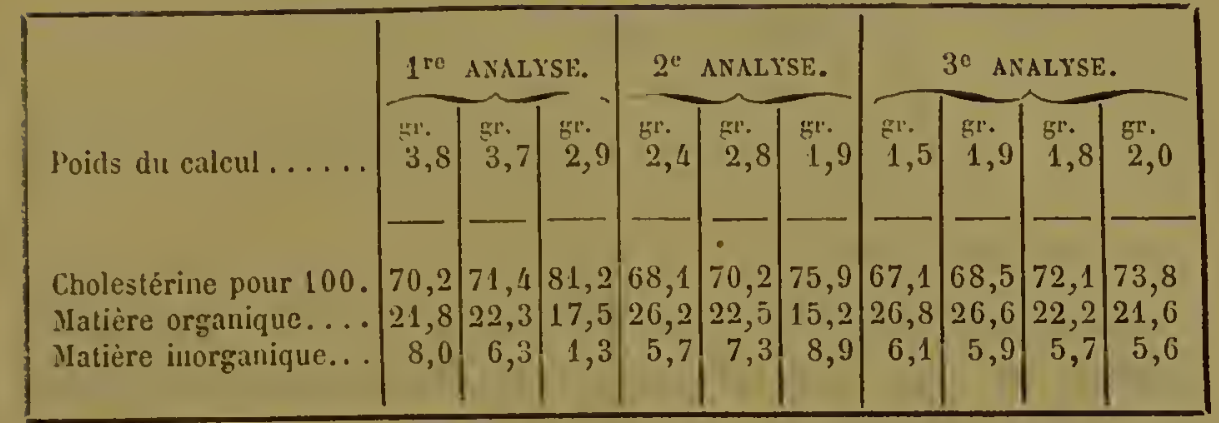

Les résultats obtenus permettent, il me semble, de tirer les conclusions suivantes: Presque toujours les calculs qui se trouvent dans la mème vésicule sont de formation simultanée ; cette donnee chimique est contrôlée par un résultat physique; ces calculs ont en clfet tous le mème poids. Lorsque les calculs se sunt formés à des périodes viriablcs, leur poids est différent, et ce sont ceux qui ont des poids semblables qui possèdent également la mème composition chimique.

Une seconde question que je me suis posée est la suivante.: La composition chimique du calcul est-elle la mème, alors que l'aspect physique ne fait pas voir des couches de nature différente?

En isolant, à l'aide du canif, les parties externes des parties centrales, j'ai pu constater que, sauf de rares exceptions, les parties cxtérieures du calcul étaient plus riches en cholestérine que les partics centralcs. Le noyau ou pour mieux dire la partie cenIrale était toujours la partie la plus riche en sels inorganiques : 19 fois sur 19 analyses c'ćtait le cas. Le fait cst moins vrai pour les calculs qui semblent formés de cholestérine pure, comme le fait voir l'analyse suivante d'un calcul presque blanc qui pesait $6^{3 \mathrm{r}}, 4$.

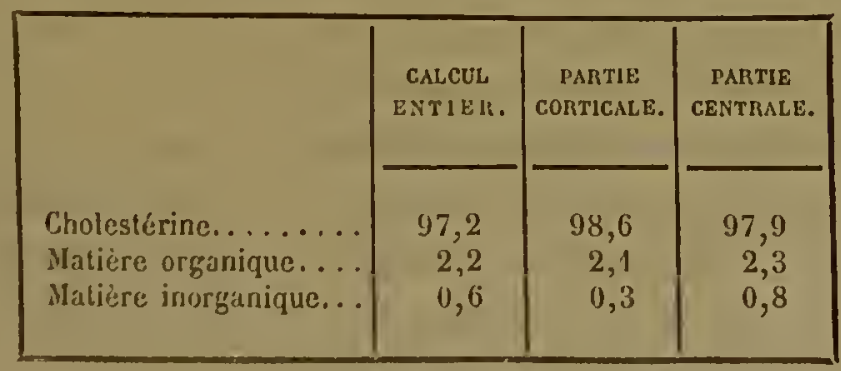

Si l'on se rapportéa ce que nous avons dit de la composition 
des cendres, on ne peut s'empecher de croire qu'il y a de fortes probabilités pour admettre que ce sont les composés insolubles de pigments, des acides hiliaires ou gras, qui forment bien souvent le centre de cristallisation ou le noyiu.

Les nombreux calculs que j'avais à ma disposition m'ont engigé à étudier l'action que les alcalis faibles ì la dose de 1 à 5 millièmes auraient sur eux à la température de 40 degrés. Les médecins s'accordent assez généralement à reconnaître l'influence heureuse que la médication alcaline exerce sur la marche des affections calculeuses de la vésicule biliaire; on n'a qu'à consulter à cet égard l'ouvrage si remarquable du docteur Villemin, médecininspecteur des eaux de Vichy. Cette aetion s'explique difficilement, car la cholestérine est insoluble dans les alealis et n'est soluble qu'en quantité très-faible dans les savons et les sels alealins des acides biliaires. Aussi j’ai remarqué que les calculs de la $1^{\mathrm{re}}, 2^{\mathrm{e}}$ el $5^{\mathrm{e}}$ classe, c'est-a-dire ccux où la cholestérine est ì la surfaee, n’ont jamais rien perdu de leur poids dans les solutions alcalines faibles, $m$ m au bout de trois mois. Il n'en était pas de même des calculs de la $3^{\mathrm{e}}, 4^{\mathrm{e}} \mathrm{ct} 6^{\mathrm{e}}$ classe ; ces dernier's surtout étaient modifiés le plus profondément. Voici ce que l'on observe: La matière colorante qui forme conme le cinient du calcul se dissout peu à peu; la périphérie du calcul est corrodée el la cholestérine se détaehe en petites pailletles qui nagent dans le liquide; l'agitation, le renouvellement du liquide alcalin favorisent cette action, mais elle est dans tous les cas très-faible. Ainsi un calcul de la $3^{\mathrm{e}}$ classe pesant $3^{\mathrm{gr}}, 7$ a perdu au bout de trois mois $0^{\mathrm{gr}}, 18$ dans la solution alcaline à 3 pour 1000 , alors qu'un calcul $d^{\prime}$ un poids de $3^{\mathrm{gr}}, 4$ placé dans l'eau distillée dans les mèmes conditions de température et de temps n'a perdu que 0,04 .

Cette expérience semble donner la clef de la manière d'agir des alcalis ; sous leur influcnee l'économie (le fait a été démontré) sécrète des humeurs plus alcalincs: les composés organiques à base de chaux sont alors transformés en composés alcalins solubles qui ne se déposent pas; le calcul cesse done de s'accroittre; le calcul déjà formé peut se désagréger petit à petit par l'action des alcalis sur le ciment formé par les matières colorantes. 
SLR LA COMPOSITION DES CALCULS BILIAIRES HUMAINS.

Si celte hypothese est vraic, les alculs de cholestérine pure doivent etre les plus volumineux, car, une fois formés, le nouveau milieu u'a plus d'influence sur cux; pour les autres on conçoit que la sécrétion biliaire peut clıanger à un moment donné et redissoudre le précipité qui s'est formé à une autre période. Voici ce que nous enseignent nos analyses.

D'une parl, les 3920 calculs qui pesaient moins que 0,1 n'étaient jamais formés par de la cholestérine pure; d'autre part, les calculs les plus volumineux étaient les plus riches en cholestérine; ce sont en effet les calculs de la première et de la deuxième classe qui pesaient de 6 à 14 grammes.

Quelques mots encore sur les causes qui peuvent amener la formation des calculs.

L'influence d'un noyau provenant soit de l'extérieur, soit de l'intérieur, est admise sans conteste pour les calculs vésicaux. Tout le monde connait l'influence du fil dans le procédé de Garrod pour la recherche de l'acide urique dans le sang des gou!tcux.

Sans la présence du fil, l'acide urique ne se disposerait pas à l'état cristallin, mais à l'état pulvérulent, et mème it pourrait rester en solution un temps assez long pour être éliminé au dehors à l'état soluble; le mucus, les savons calcaires des matières colorantes des acides biliaires, etc., remplissent certainement dans la vésicule le rôle du fil de Garrod. Or un noyau, un centre d'attraction étant donné, on comprest qu'une humeur qui sans ce corps étranger n'aurait pas donné de dépôt, laisse précipiter de la cholestérine.

Mais ce u'est pas sur ces cas que je veux appeler l'attention. Une solution saturée d'acide urique cristallise mème sans la présence d'un corps étranger lorsque la température s'abaisse; on peut admettre ipue le foie biliaire tire du sang un liquide plus riclıe en cholestérine que dans les conditions nornales; cet excès de cholestérine se trouvant dans 11 milieu dont la composilion varie d'un instant a l'autre, pent se precipiter et jouer alors luiwène le roble de centre d'attraction ou decristallisation. Cie morle deformalion de calculs roit rexister a mon avis, et je me base sur les faits suivants pont biayer relte maniere de voil. 
Les femmes, ct en général les persounes qui ménent une vic sédenlaire, sont fréquenment alleintes de coliques hépatiques; or, chez toutes ces personnes les oxydations ne se fout pas avec leur intensité normalc. N'est-il pas curieux de voir que chez des animaux soumis à l'influence de corps que l'on dit agir comme désoxydants (phosphore, arsenic, antimoine, etc.), le sang devient plus riche en cholestérine? Ce fait ressort d'expériences inédites que je publierai sous peu.

Si inaintenant nous admettons la théorie de Mialhe sur l'influence que les alcalis exercent sur les combustions interorganiques, nous expliquerions peut-être d'une manière th sùre les résultats obtenus par la médication alcaline, ou comprendrait que les calculs cessent de s'accroître; et si nous admettons l'action physique corrélative dont nous avons donné des nreuves, on entrevoit la possibilité de l'élimination de calculs déjà formés.

Un fait du mème ordre est le suivant; je le recommande spécialement à l'attention des médecins aliénistes: c'est dans les asiles que j'ai pu me procurer' à la fois, non-seulement les calculs les plus nombreux, mais encore les calculs les plus riches en cholestérine. Le repos, l'inaclivité souvent imposés à ces malheureux sont-ils la cause de ces dépôts? ou bien devons-nons admettre que le système nerveux malide sécrète plus de cholestérine? Celte dernière scrait-elle, comme le veut Austin Flint, un produit de la désassimilation de la substance cérébrale, el ces observations confirmeraient-elles l'existence de l'étal pathologique que cet auteur appelle la cholestérémie?

Je ne puis me prononcer ; je me borlle à faire remarguer que, si le premier cas est vrai, on devra trouver dans les maisons do correction, centrales, etc., la móme proportion de calculs que dans les asiles d'aliénés. Jo me contente de rapporter le finit et d'appeler sur lui l'attention des médecins qui sont dans le cas de le vérifier. 


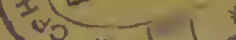

PL. I
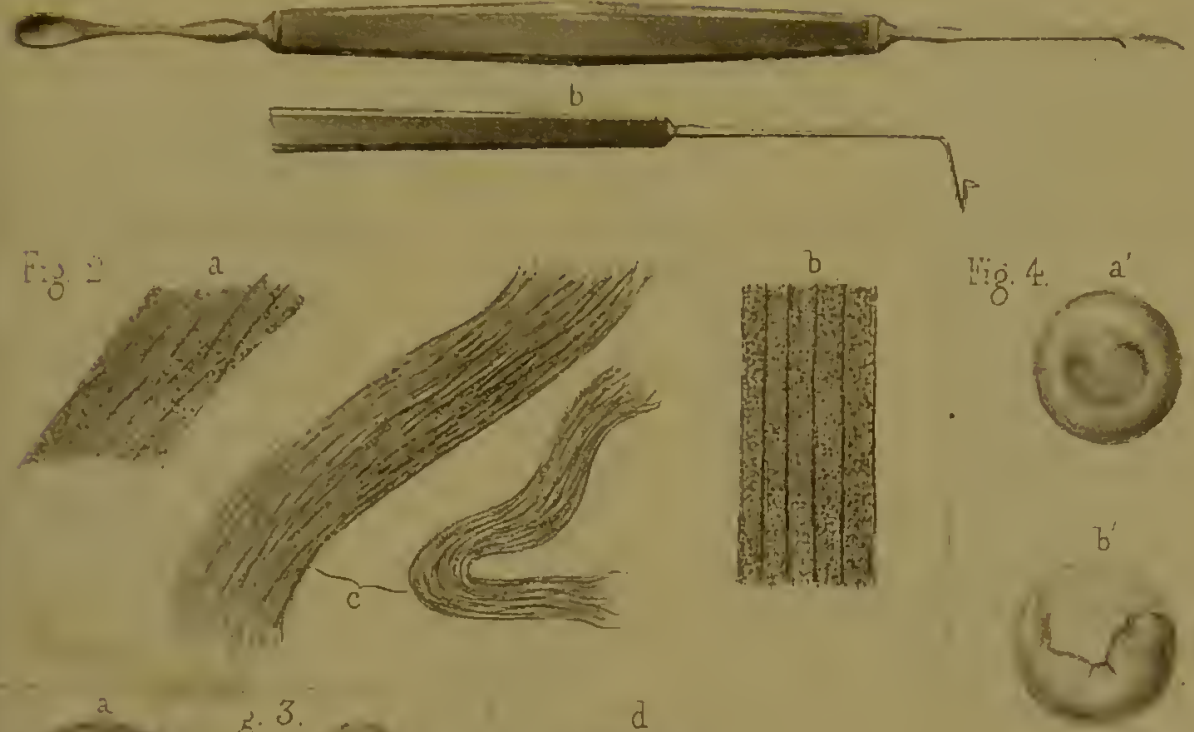

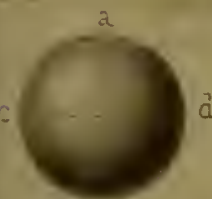

$A^{b}$

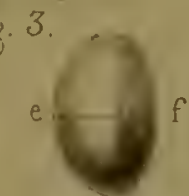

B

I. 5
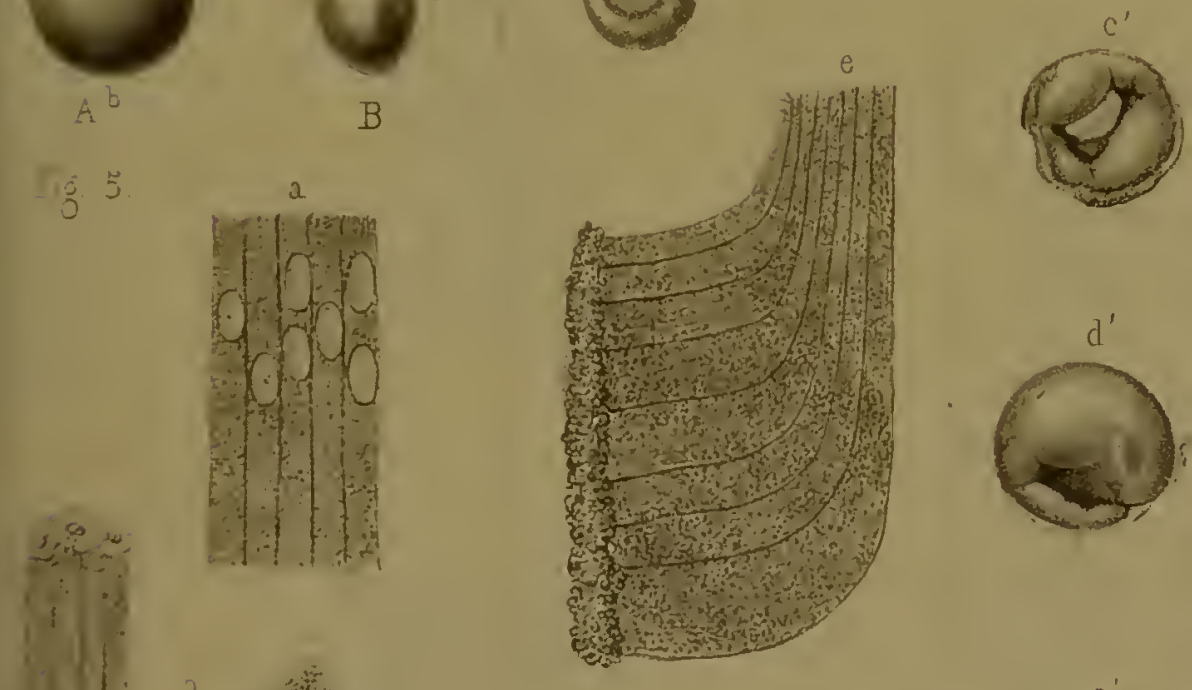

$1: \begin{cases}0 & 3 \\ 1-1,160 & -3\end{cases}$

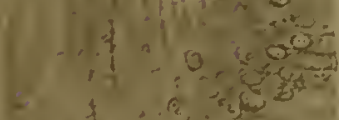
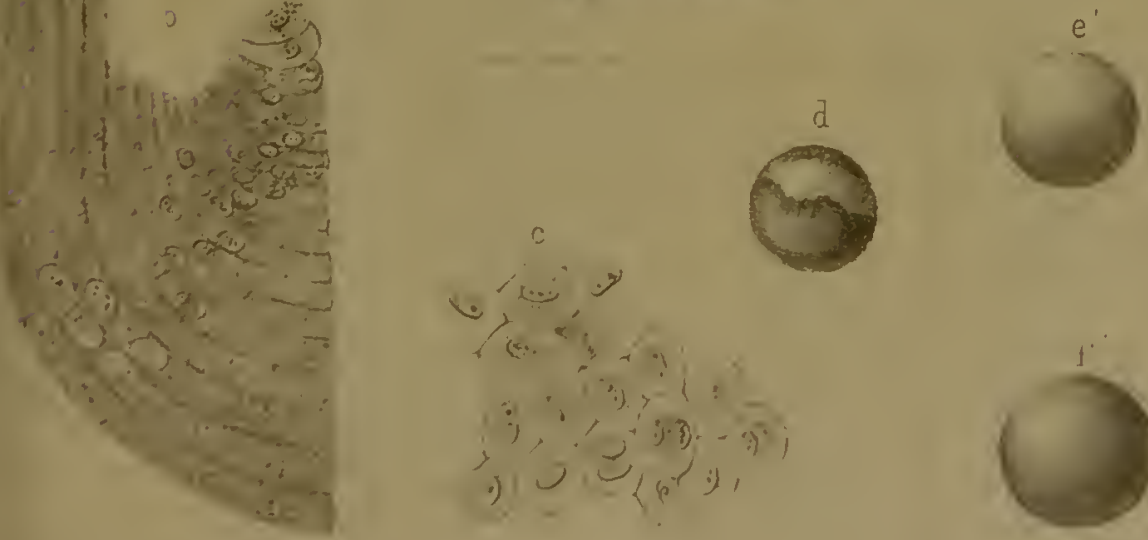

;

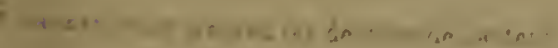
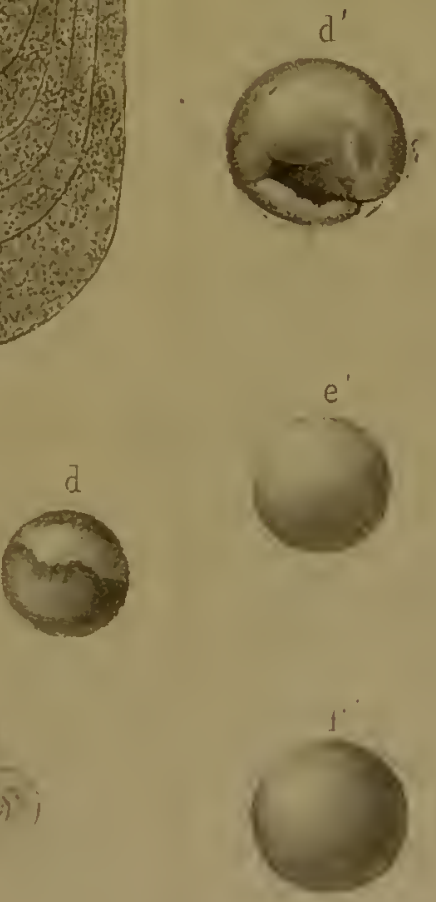

Regenciation du Coristallin. 




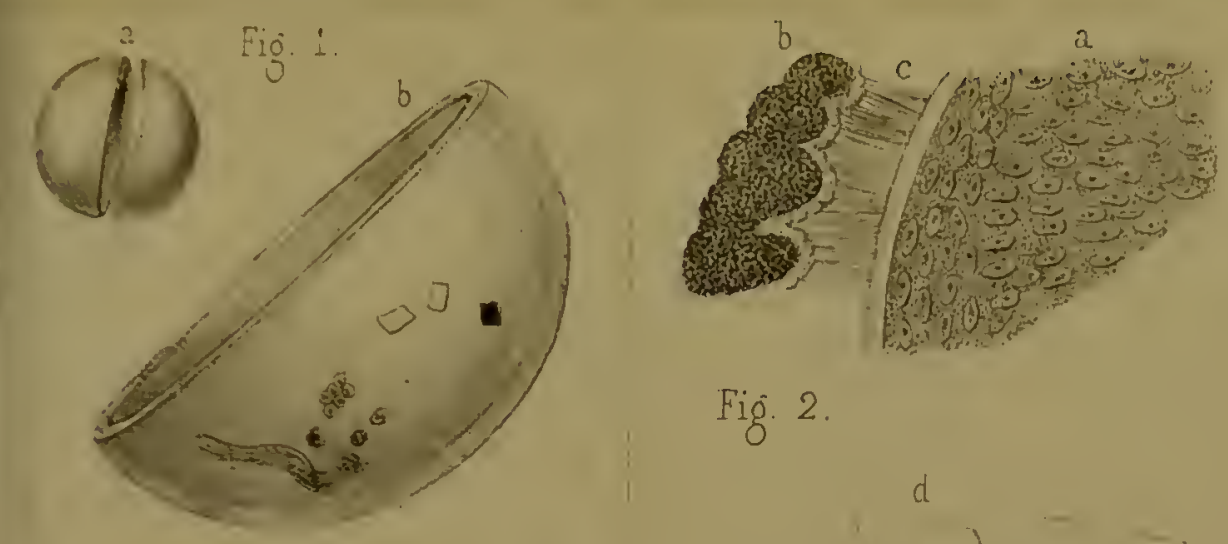

Fig. 2 .
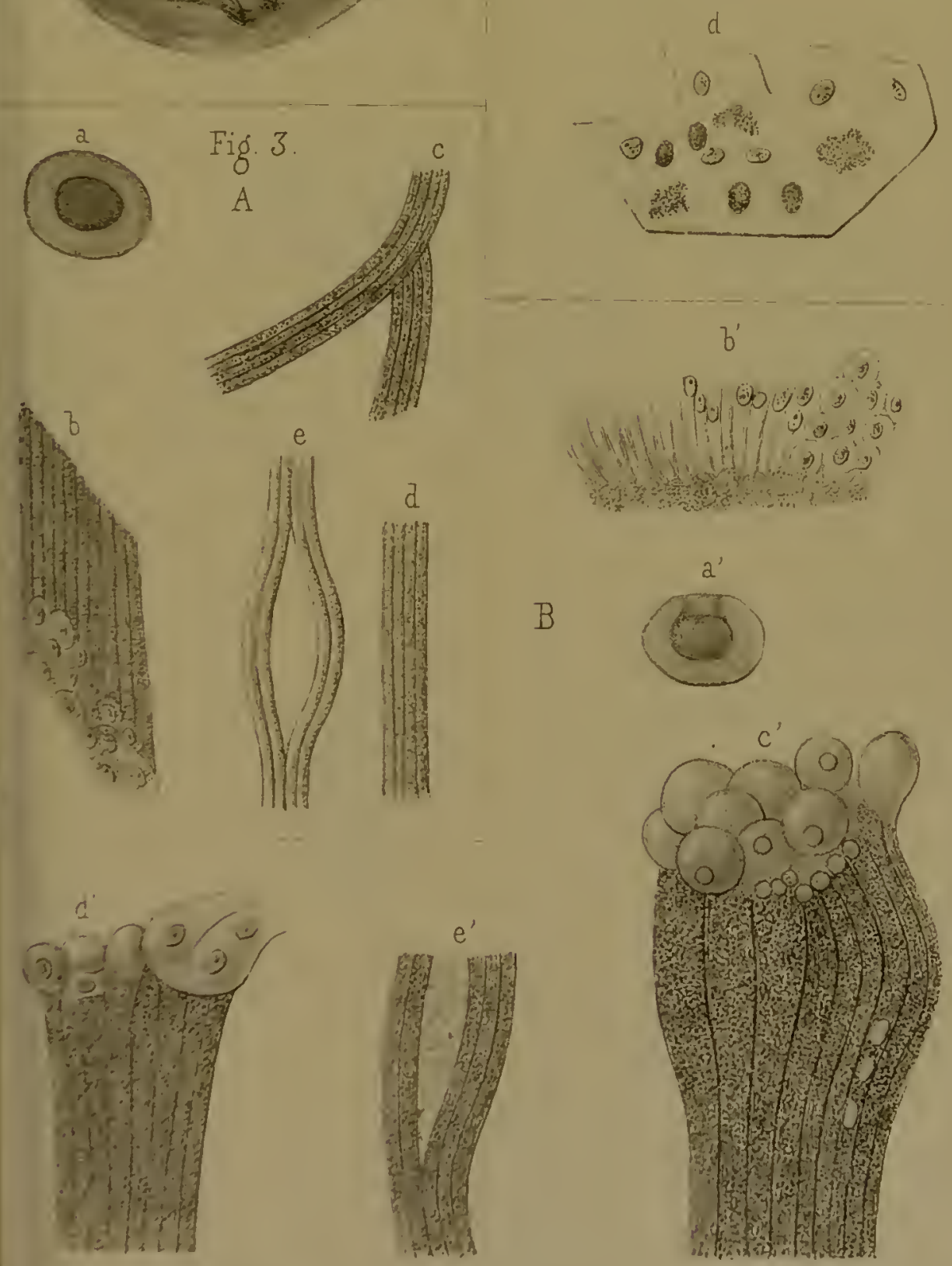

Irop Becquet a Paris.

Régénération du Cristallin. 


